\title{
Thermoelectric \\ Polymer-cellulose \\ Composite Aerogels
}

Shaobo Han
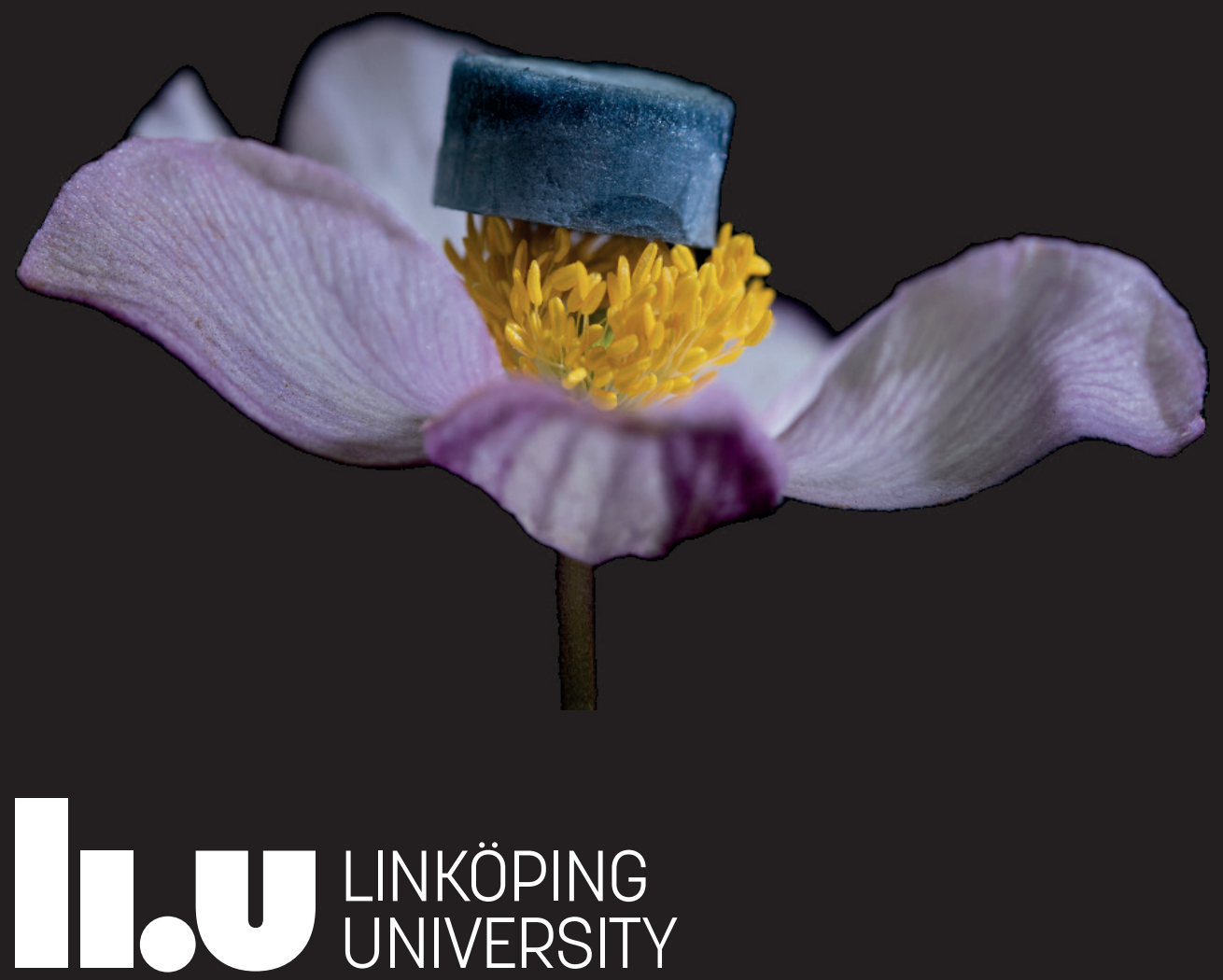


\title{
Thermoelectric polymer-cellulose composite aerogels
}

\author{
Shaobo Han
}

Department of Science and Technology

Linköping University, Sweden

2019 Norrköping 


\section{Thermoelectric polymer-cellulose composite aerogels}

(C) Shaobo Han, 2019

During the course of the research underlying this thesis, Shaobo Han was enrolled in Agora Materiae, a multidisciplinary doctoral program at Linköping University, Sweden.

Printed by LiU-Tryck, Linköping, Sweden, 2019

ISBN 978-91-7929-967-5

ISSN 0345-7524 


\begin{abstract}
Thermoelectric materials are scrutinized as energy materials and sensing materials. Indeed, they convert thermal energy into electrical energy. In addition, those materials are actively sensitive to a temperature modification through the generation of an electric signal. Organic thermoelectric (OTE) materials are complementary to inorganic thermoelectric materials, as they possess unique properties such as solution processing, ionic conductivity, flexibility, and softness. While thin-film OTE materials have been widely studied because they are easily manufactured by various coating techniques, little is done in the creation of three-dimensional morphologies of OTE materials; which is important to develop large temperature gradients.

Cellulose is the most abundant biopolymer on the planet. Recently, the applications of cellulose are not only limited in making papers but also in electronics as the cellulose provide 3-D microstructures and mechanical strength. One promising approach to make 3-D OTE bulks is using cellulose as scaffold because of their properties of relatively high mechanical strength, water processability and environmentally friendly performance.

The aims of the thesis have been to enlarge the applications of an OTE material poly $(3,4-$ ethylenedioxythiophene) (PEDOT), with an approach of making 3-D aerogels composite with nanofibrillated cellulose (NFC), in two main areas: (1) multi-parameter sensors and (2) solar vapor generators. In the first application, we demonstrate that the new thermoelectric aerogel responds independently to pressure $\mathrm{P}$, temperature $\mathrm{T}$ and humidity RH. Hence, when it is submitted to the three stresses (T, P, RH), the electrical characterization of the material enables to measure the three parameters without crosstalking effects. Thermoelectric aerogels are foreseen as active materials in electronic skins and robotics. In the second application, the conducting polymer aerogels are employed as solar absorbers to convert solar energy into heat and significantly increased the water evaporation rate. The IR absorption is efficient because of the free-electron in the conducting polymer PEDOT nano-aggregates. Because of the low cost of those materials and the water stability of the crosslinked aerogels, they could be of importance for water desalination.
\end{abstract}




\section{Populär Sammanfattning}

Termoelektriska material har utvärderats som energi- och sensormaterial. Som energimaterial har de studerats som ett sätt att transformera termisk energi till elektrisk energi, och har använts för kylnings- och uppvärmningsapplikationer. Som sensormaterial kan de känna av temperatur eller temperaturskillnader och tillhandahåller elektriska signaler. Organiska termoelektriska (OTE) material, det vill säga kolbaserade termoelektriska material, är komplementära till inorganiska termoelektriska material eftersom de har unika egenskaper så som processbarhet i lösningsform, jonisk ledningsförmåga, böjbarhet, och mjukhet. Tunna filmer av OTE-material har vida studerats eftersom de är lätta att tillverka via olika beläggningsmetoder, men tredimensionella strukturer är till stor del ett outforskat område och är viktigt för att uppnå stora temperaturgradienter.

Cellulosa är ett billigt material som utgör den vanligaste biopolymeren på vår planet. Nyligen så har applikationerna för cellulosa sträckt sig bortom papperstillverkning och används nu även inom elektronik för att förse 3D-mikrostrukturer och mekanisk styrka. En lovande metod för att tillverka 3D-strukturer av OTE-material är genom att använda cellulosanätverk på grund av dess relativt höga mekaniska styrka, processbarhet $\mathrm{i}$ vattenlösningar och dess miljövänlighet.

Syftet med denna avhandling har varit att bredda applikationerna för OTE-materialet poly(3,4-ethylenedioxythiophene) (PEDOT), genom att tillverka 3D aerogelkompositer med nanofibrillerad cellulosa (NFC). Detta har gjorts inom två områden: (1) Multiparameter-sensorer och (2) solar vapor generators. För den första applikationen så demonstrerar vi att de nya termoelektriska aerogelerna har oberoende signaler från tryck, temperatur och relativ fuktighet. Det vill säga att när materialet utsätts för dessa stimuli så kan signalerna som genereras urskiljas av utan överhörning. De termoelektriska aerogelena förutses bli användbara inom områden så som elektronisk hud och robotik. För den andra applikationen används de elektriskt ledande aerogelena för att absorbera solljus för att omvandla solenergi till värme vilket kan öka förångningshastigheten hos vatten. Absorptionen i IR-området är effektivt eftersom de rörliga elektronerna i den ledande polymeren nano-aggregerar. På grund av den låga kostnaden hos dessa material och våtstabiliteten hos korslänkade aerogeler kan dessa material tänkas användas för vattenavsaltning. 


\section{Acknowledgements}

First and foremost, I would like to express my deep gratitude to Xavier Crispin and Simone Fabiano, my academic supervisors, for their patient guidance, great help, and enthusiastic encouragement.

My grateful thanks are also extended to many of other seniors, colleagues, and friends. This thesis would not have been a reality without your help and support. I must give many thanks to:

Magnus Berggren, Igor Zozoulenko, Isak Engquist, Daniel Simon, Eleni Stavrinidou, Klas Tybrandt, Eric Glowacki, Mikhail Vagin, Viktor Gueskine, and Jaywant Phopase, for delivering your knowledge and experiences.

Fei Jiao, my mentor, for showing me how to start a new life in Sweden and how the lab works.

Zia Ullah Khan, my technique teacher, for showing me how to start a Ph.D study and what a superior Ph.D looks like.

Anna Håkansson, for collaborations, for ordering chemicals, and most importantly, for being my first Swedish friend.

Gábor Méhes, Hjalmar Granberg, Jesper Edberg, Lars Berglund, Lars Granlöf, Miriam Huerta, Nara Kim, and Naveed UL Hassan Alvi, for collaborations and sharing many of your interesting ideas.

Chiyuan Yang, Dan Zhao, Deyu Tu, Fareed Ahmed, Ioannis Petsagkourakis, Jennifer Gerasimov, Kosala Wijeratne, Suhao Wang, Shangzhi Chen, Tero-Petri Ruoko, Tobias Abrahamsson, and Ujwala Ail, for many interesting discussions and kind help.

Many other friends from LOE, Ahmadou Ly, Andrea Grimoldi, Anton Volkov, Arman Molaei, Dagmawi Belaineh Yilma, David Poxson, Donata Iandolo, Donghyun Kim, Eliot Gomez, Evan Kang, Evangelia Mitraka, Fatima Nadia Ajjan, Felipe Franco Gonzalez, Gang Wang, Gwennael Dufil, Hamid Ghorbani Shiraz, Hengda Sun, Hongli Yang, Iwona Bernacka Wojcik, Johannes Gladisch, Kai Xu, Lixin Mo, Maciej Gryszel, Maria Seitanidou, Marie Jakesova, Mert Vural, Mehmet Girayhan Say, Mina Shiran Chaharsoughi, Nitin Shriram Wadnerkar, Penghui Ding, Robert Brooke, Renbo Wei, Samim Sardar, Samuel Lienemann, Sergi Riera-Galindo, Silan Zhang, Skomantas 
Puzinas, Vasileios Oikonomou, William Armando Munoz, Xiane Li, Xianjie Liu, Xiaoyan Zhou, Xenofon Strakosas, Ziyauddin Khan, and all other LOE numbers, for the jokes, cakes, food, fika, and all the fun time that we shared.

The administrative team, Åsa Wallhagen, Katarina Swanberg, Lesley G Bornhöft, Kattis Nordlund, and Jenny Joensuu, for your help on many activities beside research.

The lab management team, Las Gustavsson, Meysam Karami Rad, Thomas Karlsson, Patrik Eriksson, and Anna Malmström, for your hard work to keep the lab running and in order.

The heads of Agora Marteriae, Per-Olof Holtz and Caroline Brommesson, for organizing fantastic study activities.

My father, mother, sister, and nephew, for your encouragement, support, and love.

Finally, I would like to thank my wife, my love, Canyan Che. There is so much I want to say but so little that can be expressed. You created a super comfortable home for us and always be my audience. Thank you for your support and love.

Thanks to everyone I have met along the way. 


\section{List of publications}

Paper 1: Effect of (3-Glycidyloxypropyl)Trimethoxysilane (GOPS) on the Electrical Properties of PEDOT:PSS Films

Anna Håkansson, Shaobo Han, Suhao Wang, Jun Lu, Slawomir Braun, Mats Fahlman, Magnus Berggren, Xavier Crispin, Simone Fabiano*, Journal of Polymer Science, Part B: Polymer Physics, 2017, 55, 814-820

Contribution: I contributed to part of the experiment design (20\%), performed part of the experimental work (30\%), and contributed to the editing of the manuscript.

Paper 2: Thermoelectric Polymer Aerogels for Pressure-Temperature Sensing Applications

Shaobo Han, Fei Jiao, Zia Ullah Khan, Jesper Edberg, Simone Fabiano, and Xavier Crispin*, Advanced Functional Materials, 2017, 1703549

Contribution: I contributed to the experiment design, performed most of the experimental work, wrote the first draft and contributed to the editing of the final manuscript.

Paper 3: A Multiparameter Pressure-Temperature-Humidity Sensor Based on Mixed Ionic-Electronic Cellulose Aerogels

Shaobo Han, Naveed Ul Hassan Alvi, Lars Granlöf, Hjalmar Granberg, Magnus Berggren, Simone Fabiano, and Xavier Crispin*, Advanced Science, 2019, 1802128

Contribution: I contributed to the experiment design, performed most of the experimental work, wrote the first draft and contributed to the editing of the final manuscript. 
Paper 4: Cellulose-Conducting Polymer Aerogels for Efficient Solar Steam Generation

Shaobo Han, Tero-Petri Ruoko, Johannes Gladisch, Xavier Crispin, Simone Fabiano, Manuscript

Contribution: I contributed to the experiment design, performed most of the experimental work, and wrote the first draft of the manuscript. 


\section{Table of Contents}

\section{Part I: Background}

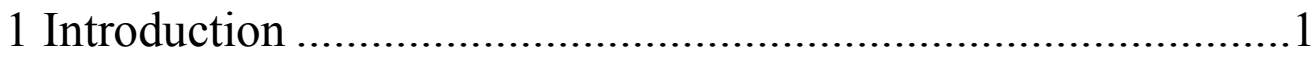

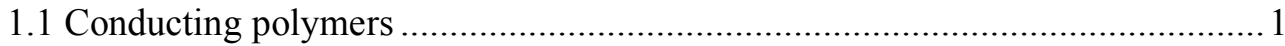

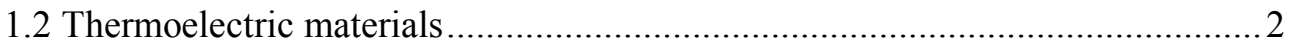

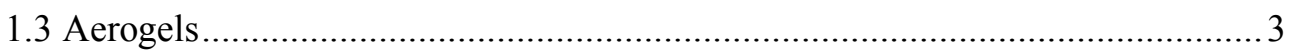

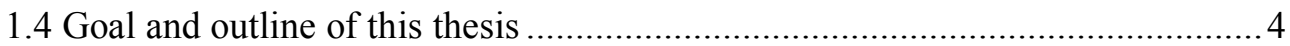

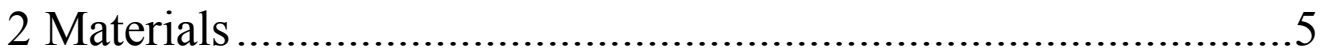

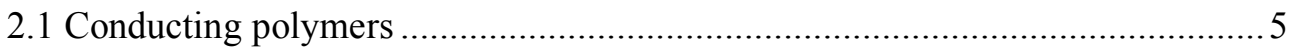

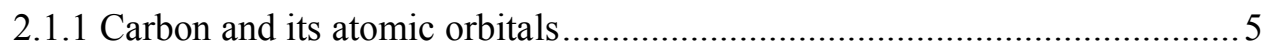

2.1.2 Electronic structure of carbon-based molecules ....................................... 8

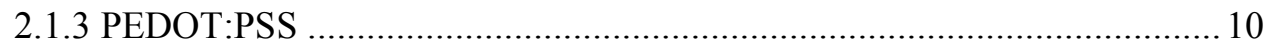

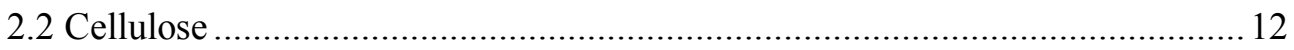

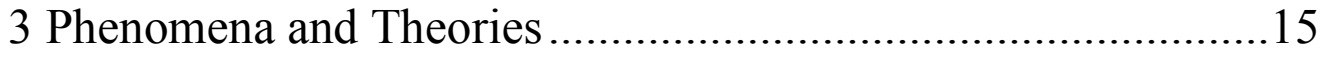

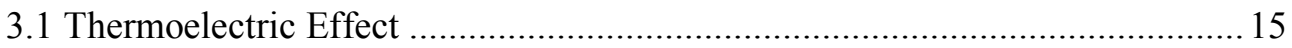

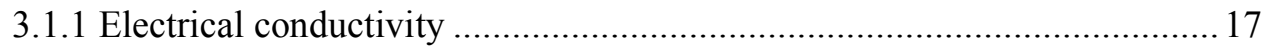

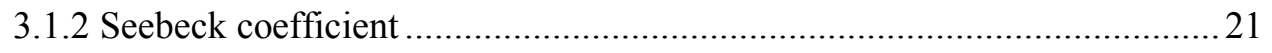

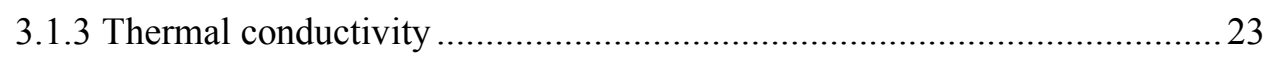

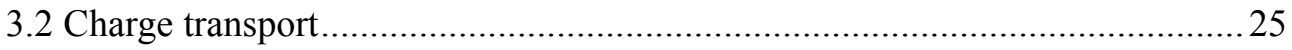

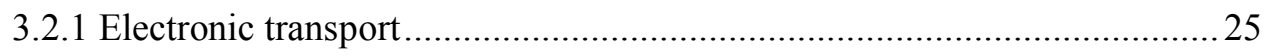

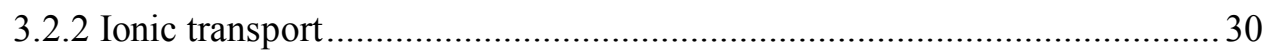

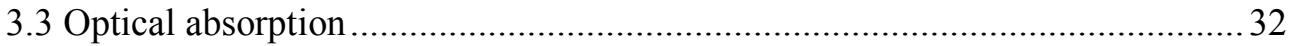

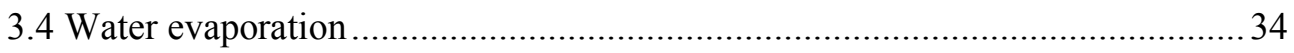

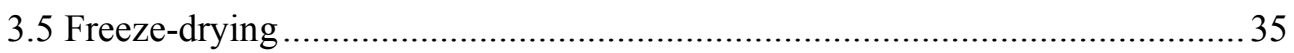

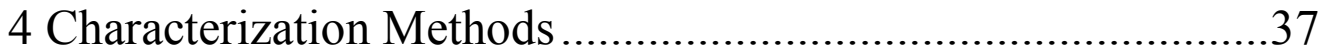

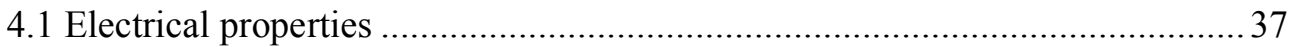

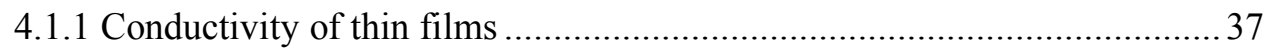


4.1.2 Resistance and Seebeck coefficient of aerogels......................................... 38

4.2 Scanning Electron Microscope (SEM) ………............................................ 40

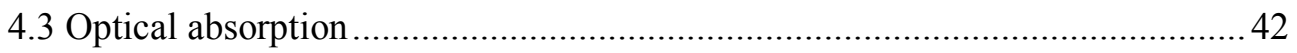

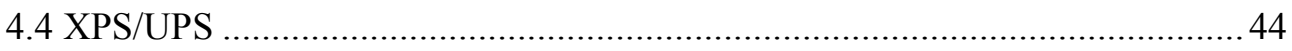

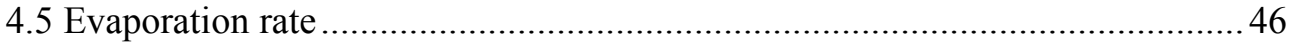

5 Conclusion and Outlook .................................................49

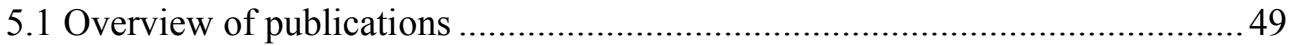

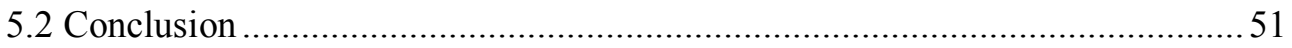

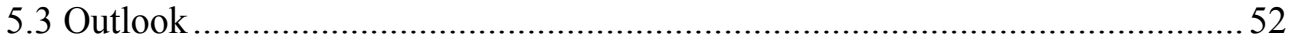

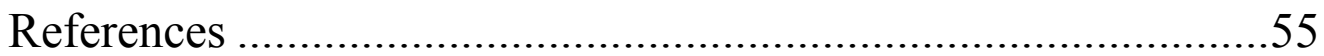

\section{Part II: Publications}


Part I

Background 



\section{1}

\section{Introduction}

\subsection{Conducting polymers}

Conjugated polymers are usually based on aromatic monomers coupled to each other via $\pi$-electrons delocalized along the backbone of the polymer chain. Conjugated polymers can be either semiconducting or conducting. Electronic charge carriers can be transported in an electrical field within the polymer chains and jump from one to the nearest chain to achieve multiple levels of electrical conductivity like insulators, semiconductors or poor metals. Conducting polymers are widely investigated mostly in thin (thickness $<10 \mu \mathrm{m}$ ) or ultra-thin films (thickness $<0.1 \mu \mathrm{m}$ ) in many areas such as electronics (transistors, diodes) and optoelectronics (solar cells, light emitting diodes, photodetectors) ${ }^{1-4}$. Depositing polymer thin films on a substrate can easily be done from solution by means of various coating techniques. Thin films have typically reduced number of defects compared with thick films that display problems such as cracks and delamination. Moreover, depending on the manufacturing technique, thin films show a preferential morphology with alignment of the chains relative to the substrate. Therefore, the electrical conductivities of thin films are typically higher than thick films. Going beyond thin-film technologies (thickness $>10 \mu \mathrm{m}$ ) is an exciting research area, where new applications could be reached, such as supercapacitors, batteries, thermoelectrics, and electronic skins ${ }^{5-7}$. One strategy to bring conducting polymers to thick film is to combine them with soft $3 \mathrm{D}$ scaffolds, such as nanofibers of cellulose ${ }^{8}$. 


\subsection{Thermoelectric materials}

The distinction between various classes of materials, e.g. insulators, semiconductors and conductors, lies in the occupation of the energy space by the electrons, which is defined by the electronic density of state. When a material is subjected to a temperature gradient, the occupation of the density of state is different on the hot and cold side of the material. This results in a diffusion of charge carriers (holes and electrons) from the hot to the cold side. In most of the materials, electrons and holes accumulate in a non-equal fashion at the cold side. An electrical potential will be therefore generated between two ends of a homogeneous material (e.g. semiconductor) subjected to the temperature gradient. The ratio between the electric potential and the temperature gradient defines the Seebeck coefficient, which is an intrinsic material property ${ }^{9-11}$.

All materials display thermoelectric (TE) properties. However, most of the materials cannot be used as TE materials because of at least one of these three reasons: (i) the electrical conductivity is so low that the thermodiffusion of charge carriers provides negligible electrical current and power. (ii) The ability of creating an electric potential is so low that it provides almost no potential even for a considerable temperature gradient. (iii) The thermal conductivity is so high that the temperature gradient cannot be holden. That is, only the materials that have high electrical conductivity, high Seebeck coefficient and low thermal conductivity are employed as TE materials. In other words, an ideal TE material is often described as an 'electron crystal/phonon glass'. Inorganic semiconductors are widely studied and used as TE materials because they are generally electron crystals. By reducing the thermal conductivity, many inorganic TE materials have been discovered and optimized. Alternatively, conducting polymers as an example of organic (carbon based) materials have been recently investigated as another class of TE materials for applications at room temperature, due to their intrinsically low thermal conductivity, high flexibility, solution processability and high abundance of their atomic elements.

The applications of TE materials (organic and inorganic) include thermoelectric generators which transform thermal energy directly into electrical energy (based on the Seebeck effect), and thermoelectric coolers which pump heat when an electrical 
current is applied through (based on the Peltier effect). Thermoelectric modules are further widely used as heat flux and temperature sensors.

\subsection{Aerogels}

The first aerogel was fabricated by Sammuel Stephens Kistler in 1931, as a result of a bet with Charles Learned over who could replace the liquid in jellies with a gas without causing shrinkage of the material ${ }^{12}$. An aerogel is not a 'gel' in the technical perspective. Interestingly, the term 'gel' in aerogel originates from its precursor, the liquid gel, in which the liquid has been replaced with a gas through a proper drying process. Therefore, the resulting material has approximately identical shape and volume as the initial pricursor ${ }^{13}$. Many substances can be manufactured into aerogels such as silica, metal, metal oxides, carbon and polymers. Although the performance of aerogels made by different substances varies, the incredibly low density is a common property for aerogels, which is between 0.0011 to $0.5 \mathrm{~g} \mathrm{~cm}^{-3}$. That is because usually more than $99 \%$ of an aerogel is empty space/air due to high porosity. As a result, aerogels typically possess large specific areas ${ }^{14}$. There are different strategies to manufacture aerogels. The general way of these strategies is to create a gel composed of a network of solid particles bound together by physical or chemical bonds and with solvent imprisoned. The formation of the aerogel takes place under drying to remove the liquid precisely while maintaining the porous structures. Thus, how to dry a gel smartly without changing the structures of the solid phase is the key of making an aerogel. Aerogels are promising candidates for many applications such as thermal/acoustic insulation ${ }^{15}$, catalysis ${ }^{16}$, chemical sensors ${ }^{17}$, cleanup of oil spillage ${ }^{18}$, energy storage devices ${ }^{19}$, and solar steam generators ${ }^{20}$. The possibility to create conducting polymer aerogels has been little explored. At the beginning of the thesis work, only a few studies combined cellulose aerogel with conducting polymers ${ }^{21-23}$. 


\subsection{Goal and outline of this thesis}

The goal of this thesis is to create novel materials at the intersection between these three areas of research: aerogels, conducting polymers, and thermoelectrics. We combine thermoelectric polymers and cellulose based aerogels into new composites for two specific applications: sensing and water treatment.

Internet of Things (IoT) is an idiom defining the interactive communication of things to human through the internet. In the past decades, as an integral part of the Internet, IoT has gained great progress in many fields such as smart home, smart city, healthcare and elderly care ${ }^{24,25}$. Sensors are heavily explored in the area of IoT as the terminals, which provide basic original data to the analysis system and enable human or machine to interact between each other through a feedback loop. Typically, several specific sensors have been developed for sensing different signals. However, when the application requires many different types of data, the complexity of the sensor system leads to cumbersome manufacturing processes and thus, high cost. Hence, there is a requirement for simplifying the manufacturing of sensor systems. One goal of this thesis is to develop a multi-parameter sensor with the thermoelectric aerogels. We focus on the three key physico-chemical parameters: temperature, pressure and humidity. The goal is to find a strategy to read them independently within the same sensor material and the same sensing device.

Another challenge in our society is the shortage of drinkable soft water. As a green and sustainable technique, solar steam generation is a promising candidate for seawater desalination and wastewater purification. A key element for a solar steam generator is a porous material that is a good solar absorber to be placed on the top of salted/dirty water. Upon light illumination, the material heats up and its large surface area results in high water evaporation rate. Several porous materials, including aerogels, have been proposed $^{26-28}$. Besides the high conversion efficiency, flexibility, sustainability, and costs of materials and manufacturing processes are also important requirements. We investigate here the use of conducting polymers composited with cellulose to create aerogel absorbing the solar radiation. A thick aerogel can absorb more than $99 \%$ of the light, and the porous structure provides large specific surface area for water evaporation. 


\section{2}

\section{Materials}

\subsection{Conducting polymers}

Polymer, a word derived from the Greek words "many" (poly) and "parts" (mer), is used to describe a large molecule composed of several or many repeating subunits (monomers) connected via covalent bonds. There are many bio-polymers in nature, such as polyamides in proteins, polynucleotides in genes, and polysaccharides in celluloses. Some of these polymers are employed as important materials in daily life and industry, such as cotton, wool, silk and natural rubber. Beside these natural polymers, there are also a plenty of polymers synthesized by human, so called synthetic polymers, such as nylon, polyethylene, and polystyrene. Most of the polymers in daily life are electrically insulating, whether natural or synthetic. Polymers with electrical conductivity were not discovered until 1970s by three scientists, Alan MacDiarmid, Alan J. Heeger, and Hideki Shirakawa and therefor in 2000, they were awarded the Nobel Prize in Chemistry for their discovery of polymers that conduct electricity. The ability of polymers to be electrical insulating or conducting is governed by their electronic structure. Below, we introduce the basics to understand the electronic structure of polymers.

\subsubsection{Carbon and it's atomic orbitals}

A carbon atom has five atomic orbitals, namely $1 \mathrm{~s}, 2 \mathrm{~s}, 2 \mathrm{p}_{\mathrm{x}}, 2 \mathrm{p}_{\mathrm{y}}$, and $2 \mathrm{p}_{\mathrm{z}}$, as shown in figure 2.1a. As the sixth element, a carbon atom possesses six electrons. The 1s-orbital is always occupied by two core electrons which do not create any bond with the other atoms. In ground state configuration, the other four valence electrons occupy the $2 \mathrm{~s}-$ 
orbital (two electrons) and two of the 2p-orbitals (one electron per orbital), with one of the $2 \mathrm{p}$-orbitals unoccupied (figure $2.1 \mathrm{~b}$ ).

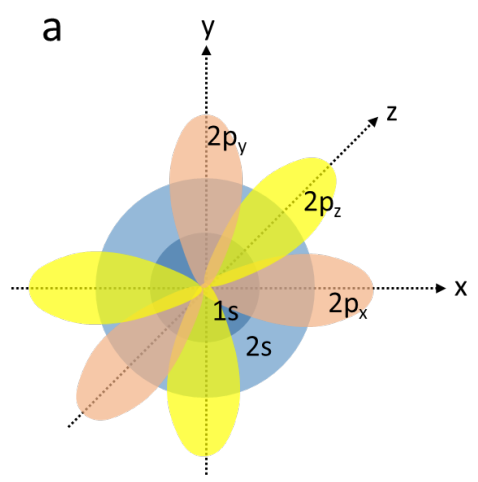

b

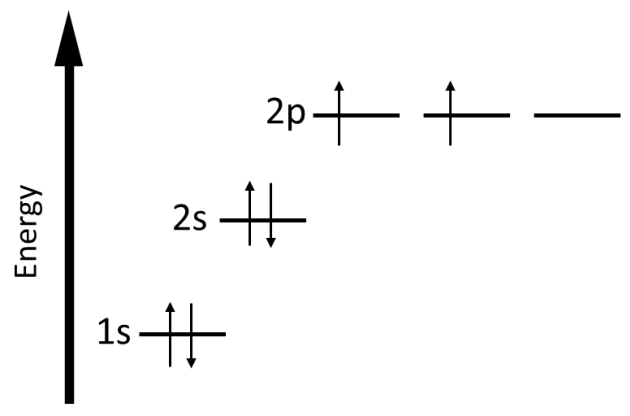

Figure 2.1 Atomic orbital of carbon and the electrons configuration in ground state.

According to the electronic configuration in the ground state, carbon has only two unpaired electrons in the $2 \mathrm{p}$-orbitals, which could be involved in bonds with maximally two other atoms. However, carbon can form four covalent bonds in some molecules such as methane $\left(\mathrm{CH}_{4}\right)$. Based on the simple electronic structure of an atom alone, it is not possible to explain the diversity of carbon-based molecules. Instead, one should consider the electric potential created by the neighboring atoms which deforms the electronic structure of the carbon atom. This phenomenon can be explained by orbital hybridization theory, as shown in Figure 2.2. According to the hybridization theory, one of the two electrons in the 2 s-orbital can be promoted to the empty $2 \mathrm{p}$-orbital, becoming an excited state. Based on this excited state, three hybridized states can be formed, as $\mathrm{sp}, \mathrm{sp}^{2}$, and $\mathrm{sp}^{3}$. The energy cost for the formation of these excited states is repaid by the creation of more bonds that stabilizing more the energy of the carbon molecule. 


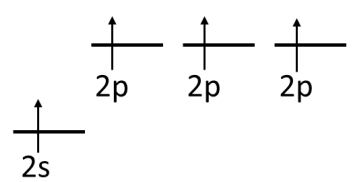

Excited state

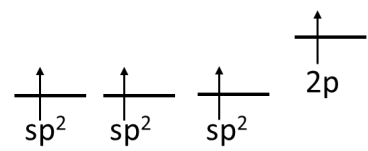

$\mathrm{sp}^{2}$-hybridization

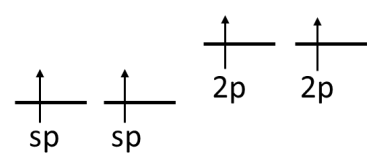

sp-hybridization

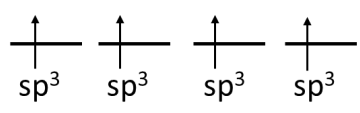

$\mathrm{sp}^{3}$-hybridization

Figure 2.2 Hybridized states of carbon.

In the sp-hybridization, the $2 \mathrm{~s}$-orbital is hybridized with one of the $2 \mathrm{p}$-orbitals, forming two equivalent sp-orbitals. In the $\mathrm{sp}^{2}$ - and $\mathrm{sp}^{3}$-hybridization, the $2 \mathrm{~s}$-orbital is then hybridized with two and three of the $2 \mathrm{p}$-orbitals, forming $\mathrm{sp}^{2}$ - and $\mathrm{sp}^{3}$-orbitals respectively. To understand how the electrons are spatially distributed, Figure 2.3 shows a naive sketch of the electronic density indicating these three types of hybridization. In the sp-hybridization, two sp-hybridized orbitals are in a line with the nuclei, while the two unhybridized $2 p$-orbitals are in a plane vertical to this line. In the $\mathrm{sp}^{2}$ hybridization, three $\mathrm{sp}^{2}$-orbitals are in a plane with the nuclei and the unhybridized $2 \mathrm{p}$-orbital is in a line vertical to this plane. There are no $\mathrm{p}$-orbitals in $\mathrm{sp}^{3}$-hybridized states, and the four $\mathrm{sp}^{3}$-orbitals tend to form a tetrahedron with an angle of $109.5^{\circ}$ between each two orbitals.

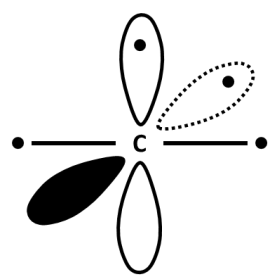

sp-hybridization

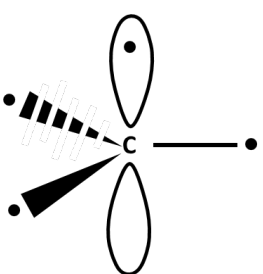

$\mathrm{sp}^{2}$-hybridization

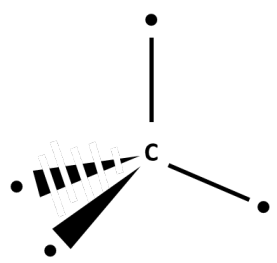

$s p^{3}$-hybridization

Figure 2.3 Electrons distribution for hybridized states of carbon. 


\subsubsection{Electronic structure of carbon-based molecules}

Through the four unpaired electrons in hybridized orbitals, carbon can form four covalent bonds with other atoms such as hydrogen atoms and other carbon atoms. A simplest molecule is methane, $\mathrm{CH}_{4}$, in which carbon form four equal bonds with four hydrogen atoms. Carbon can form different types of inter-atomic bonds with other atoms, such $\sigma$ and $\pi$ bonds. When two $\mathrm{sp}^{3}$-hybridized carbon atoms are close to each other, their orbitals overlap and form a $\sigma$-bond, as an ethane molecule $\left(\mathrm{C}_{2} \mathrm{H}_{6}\right)$ shown in Figure 2.4a. Other orbitals can also form other $\sigma$-bonds with other carbon atoms, and continuously build a long alkane chain. When two $\mathrm{sp}^{2}$-hybridized carbons are approaching each other, their hybridized orbitals overlap and form a $\sigma$-bond. Meanwhile, the two 2p-orbitals of two atoms can also overlap and form a so-called $\pi$ bond, such as the case in an ethylene molecule $\left(\mathrm{C}_{2} \mathrm{H}_{4}\right)$ shown in Figure 2.4b. Similarly, when two sp-hybridized carbon atoms are brought in proximity, their sp- orbitals overlap and form a $\sigma$-bond, the four $2 \mathrm{p}$-orbitals from these atoms overlap and form two $\pi$-bonds, as illustrated in an example of acetylene shown in Figure 2.4c.

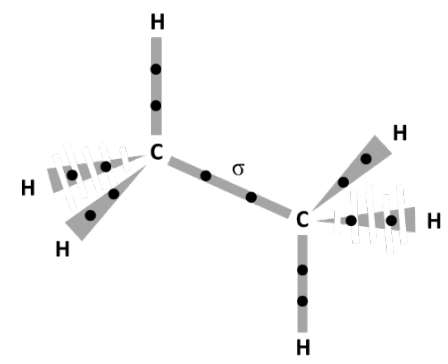

a

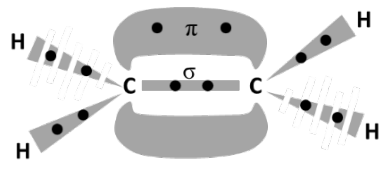

b

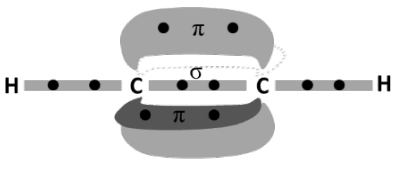

C

Figure 2.4 Molecule structure of ethane, ethylene, and acetylene.

The combination of two atomic orbitals generates two molecular orbitals, which specify the electronic configuration of the molecules. Because the wave functions of these two atomic orbitals can combine either constructively or destructively, the two molecular orbitals have different energy levels. The combination with constructive interference of the atomic orbitals has low energy level, hence the electrons in this molecular orbital are relatively stable. This molecular orbital is a bonding orbital. 
Conversely, when two atomic orbitals combine destructively, the generated molecular orbital has relatively high energy and its electrons are unstable. This molecular orbital is then defined as an antibonding orbital. Polymers that are similar to polyacetylene, having a chain with an alternation of single and double bonds, are called conjugated polymers. Since the $\pi$-bonds have a key function in electronic transport in conjugated polymers, we now focus on the electronic structure of the $\pi$-orbital and the $\pi^{*}$-orbital (the star corresponding to anti-bonding) in organic materials. Ethylene is the simplest molecule which has a $\pi$-orbital and a $\pi^{*}$-orbital separated in energy with a gap of $\sim 7$ $\mathrm{eV}$. The electronic structure of conjugated molecules and polyacetylene can be understood by considering just the $\pi$-electrons of a series of ethylene molecules attached to each other. When the molecular length gets close to infinity, the energy separation of two nearby orbitals vanishes, and these energy levels form continuous bands. The band combined with $\pi$-orbitals and $\pi^{*}$-orbitals are called valence band ( $\pi$ band) and conduction band ( $\pi^{*}$-band), respectively (Figure 2.5$)$. The energy gap $\left(E_{g}\right)$ between the highest occupied molecular orbital (HOMO) and lowest unoccupied molecular orbital (LUMO) also reduces to about $\sim 1.5 \mathrm{eV}$ in the case of polyacetylene.

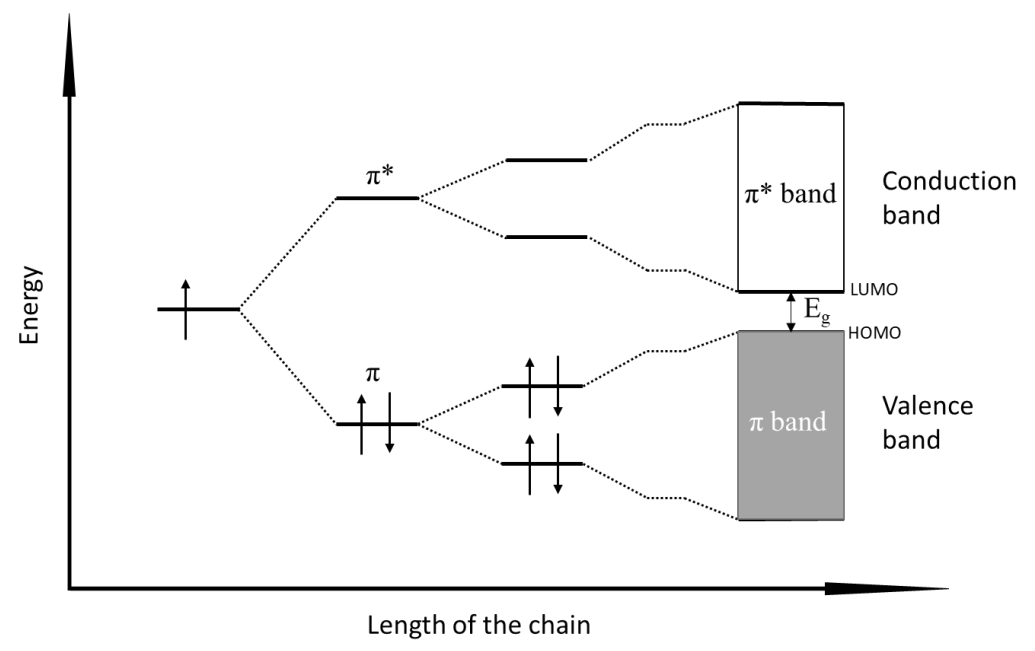

Figure 2.5 Energy band formation of polyacetylene.

In their neutral form, conjugated polymers are semiconductors and the electronic charge carriers can be transported in the $\pi$-bands without cleaving any $\sigma$-bonds 
constituting the skeleton of the polymer. However, their electrical conductivity is typically very low and close to that of typical insulators. Their band gaps between valence and conduction bands vary between $1 \mathrm{eV}$ and $4 \mathrm{eV}$. In wide band gap conjugated polymers, the charge carrier mobility increases with temperature, not mainly due to thermal excitation of the electron from the valence to the conduction band, but because transport occurs through temperature activated hopping. Nevertheless, the conductivity is still low even at high temperatures. Since the electrical conductivity is a product of mobility and charge carrier concentration, another way to increase the conductivity of semiconductors is doping, which enhances the charge carrier concentration. For conjugated polymers, these dopants act as oxidizing or reducing agents. By adding oxidizing agent, electrons transfer from polymer to the dopant, and positive charge carriers are generated on the polymer chain. This type of doping is referred to as p-doping and a p-doped polymer also possesses negative counterions to maintain the material electroneutrality. Usually, p-type polymers have low ionization potential and are easily oxidized. Reversely, n-type polymers that possess a high electron affinity are easily reduce. A polymer doped by a reducing agent has negative charge carriers on its chain and is identified as n-doped polymer with positive counterions neutralizing the polymer chains. In this thesis we have mainly employed the p-doped poly(3,4-ethylenedioxythiophene) balanced with negative counterion of polystyrene sulfonate, which is commonly abbreviated as PEDOT:PSS.

\subsubsection{PEDOT:PSS}

PEDOT is a derivative of the conjugated polymer polythiophene. It is a wildly investigated conducting polymer developed in the 1980s by Bayer AG laboratory in Germany. PEDOT can be obtained by polymerizing the EDOT monomers ${ }^{29,30}$. A cationic radical can be formed by oxidizing an EDOT monomer with an oxidant, and two cationic radicals react with each other to form a dimer by releasing a proton from each EDOT, as shown in Figure 2.6. The generated dimers can be further oxidized and react with other dimers or monomers, forming oligomers by releasing more protons. By repeating this oxidation, PEDOT chains are synthesized. The final chemical structure can be seen in Figure 2.7. 


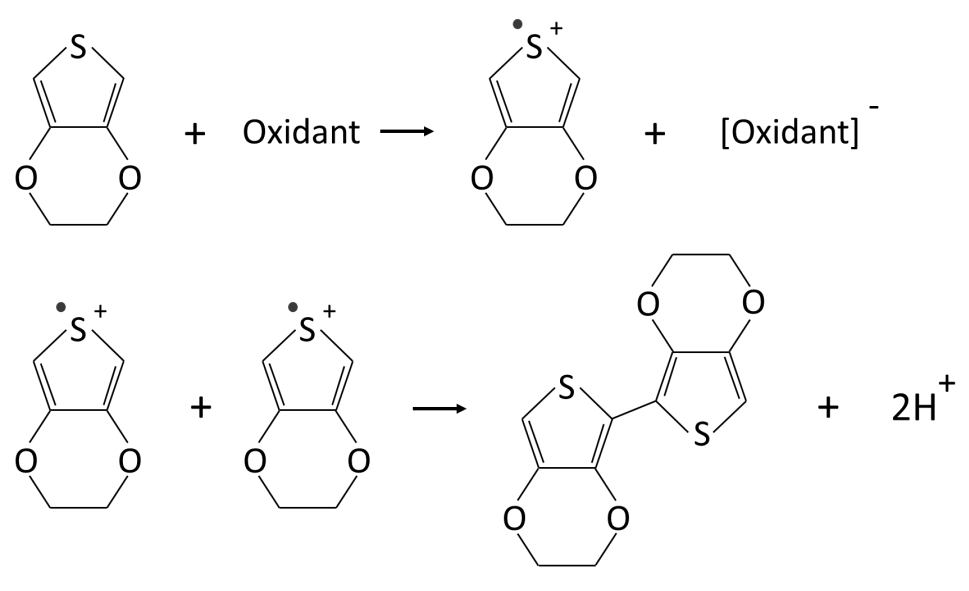

Figure 2.6 Polymerization mechanism of EDOT monomers, and PEDOT chemical structure.

Upon polymerization, the formed oligomers have a lower ionization potential than the monomer, such that they are spontaneously oxidized by the oxidant. Hence, the resulting PEDOT chains are typically p-doped with a negative counterion to keep electroneutrality. Poly(styrene sulfonate) (PSS), with the negatively charged sulfonate groups, is a wildly used counterion for neutralizing the p-doped PEDOT. The positive charge carried by the PEDOT chain changes the sequence of the bond on several units, and a polaron is formed as shown in Figure 2.7. Note that the neutral PEDOT can be obtained by de-doping the p-doped PEDOT, in other words by reducing it. But the neutral PEDOT is not much useful in electronic applications because of its low conductivity and instability in air. Indeed, it can be spontaneously p-doped by $\mathrm{O}_{2}$.

The conductivity of the blend polymer PEDOT:PSS thin film can achieve magnitude of $10^{-2} \mathrm{~S} \mathrm{~cm}^{-1}$. However, this value is still too low for many applications. The conductivity can be further increased by changing the morphology of PEDOT:PSS, with the strategy of so-called secondary doping ${ }^{31-34}$. A widely accepted explanation of the secondary doping mechanism will be further discussed in section 3.2. PEDOT:PSS that was used in this thesis was PH1000 purchased from Heraeus Equrio. It is a water dispersion with $1.3 \mathrm{wt} \%$ of PEDOT:PSS. 


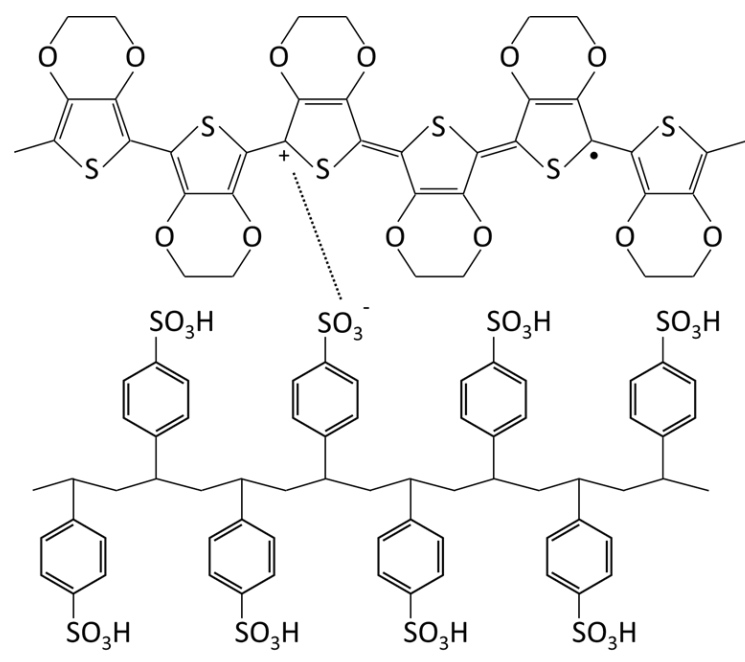

PEDOT

PSS

Figure 2.7 The chemical structure of PEDOT:PSS.

\subsection{Cellulose}

Over the past centuries, cellulose-based materials have been used as building materials, textiles ${ }^{35}$, energy source ${ }^{36,37}$, and paper $^{38-40}$. Cellulose is the most abundant component of plant biomass that can be found mostly in plant cell walls, and it is also the most abundant natural polymer on earth ${ }^{41-43}$. In 1838, French chemist Anselme Payen firstly discovered cellulose by isolating it from plant matter and determined its chemical formula. Figure 2.8 shows the general way of existence of cellulose in plants cell walls. In nature, cellulose usually appears in the form of fibers with a diameter of dozens of micrometers and a length of several millimeters. These fibers contain plenty of microand nano-fibrils composed of polysaccharide chains. As a polymer, cellulose has a long molecular chain with D-glucose as repeat units. The polymer chains of cellulose in nature consist of 7000 to 15000 glucose units. However, cellulose products, such as cellulose from wood pulp typically has 300 to 1700 glucose units, while cotton and other plant fibers as well cellulose from bacteria have chain lengths between 800 and 10000 units. Each glucose unit has three hydroxyl groups hence cellulose is very hydrophilic with the water contact angle of 20-30 degrees. Simultaneously, thanks to 
the molecular homogeneity, these hydroxyl groups on different chains have a high opportunity to be close to each other. Thus, there are plenty of hydrogen bonds among neighboring cellulose molecules. These hydrogen bonds hold the chains firmly together, therefore cellulose microfibrils have high tensile strength. Natural cellulose materials have a drawback of poor uniformity because other biopolymers, lignin and hemicellulose, are entangled between the fibers and fibrils. Lignin and hemicellulose play roles of "glue and rope" to bind the fibers or fibrils together, giving cellulose fibers high tensile strength. ${ }^{44,45}$ However, for some of the engineering applications, the feature of uniformity is required. In this case, the lignin and hemicellulose need to be removed.

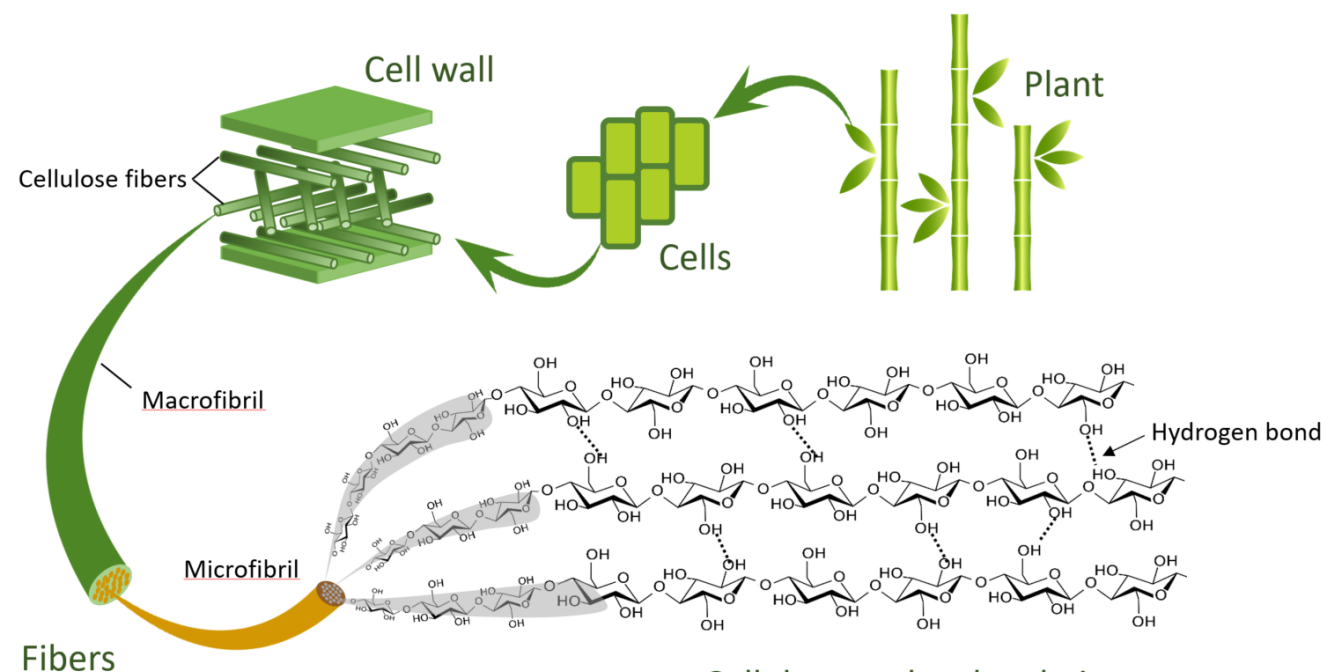

Cellulose molecular chains

Figure 2.8 A typical existence of cellulose in plants.

Nanofibers of cellulose (NFC) is composed of nano-scale cellulose fibrils with diameter of less than $20 \mathrm{~nm}$ and a wide range of lengths (typically several micrometers $)^{46}$. These tiny fibrils can be obtained by mechanical methods, such as exposing cellulose pulp to high shear forces, high-temperature, high-pressure, and high velocity impact homogenization. By this process, the large fibers are ripped into smaller nanofibrils. Because of the high energy cost of this methods to break residual binding of lignin and the interfibrillar hydrogen bonds, chemical pre-treatments are 
wildly used. Saito et al. were the first to report that 2,2,6,6-tetramethylpiperidine-1oxyl (TEMPO)-mediated oxidation can facilitate the release of NFC and, therefore, dramatically reduce the energy consumption. In the presence of a primary oxidizing agent, TEMPO catalyze the conversion of hydroxyl groups to carboxylate $\left(\mathrm{COO}^{-}\right)$ groups ${ }^{47}$. By adding these carboxylate groups, the intermolecular hydrogen bonds are broken, and the NFC chain become negatively charged. These negatively charged NFC chains have electrostatic repulsion with each other helping to form stable suspension in water. Moreover those carboxylated groups can be used to promote cross linking by other chemical reagents to form stable film of $\mathrm{NFC}^{48}$. The NFC that used in the experiments of this thesis was produced by Innventia (now RISE INNVENTIA AB) in Stockholm of Sweden. 


\section{Phenomena and Theories}

\subsection{Thermoelectric Effect}

The thermoelectric effects relate to the interplay between heat transport and electrical transport. For instance, the conversion between a temperature gradient $(\Delta \mathrm{T})$ and an electrical voltage ${ }^{49}$. There are three thermoelectric effects: the Seebeck effect, the Peltier effect, and the Thomson effect. Seebeck and Peltier effect describe two opposite processes. In 1821, physicist Thomas Johann Seebeck discovered that a compass needle could be deflected by a loop formed of two different metal wires joined in two places, with a temperature difference between these two joints. This phenomenon was later explained in terms of diffusion of charge carriers in available energy levels of the material subject to a $\Delta \mathrm{T}$. Therefore, a potential difference between the junctions is established (the Seebeck effect) and a current is then generated. A magnetic field appears around the wires crossed by an electrical current, hence, the compass needle is deflected. The conversion of the heat into electricity at the junction of different materials takes place via the Seebeck effect, as shown in Figure 3.1a. The Peltier effect was observed by Jean Peltier in 1834, which describes the reverse process of the Seebeck effect as shown in Figure 3.1b. By applying an electrical current through a junction of two different materials, heat is transported and the temperature of the junction could be either increased or decreased, depending on the materials. 

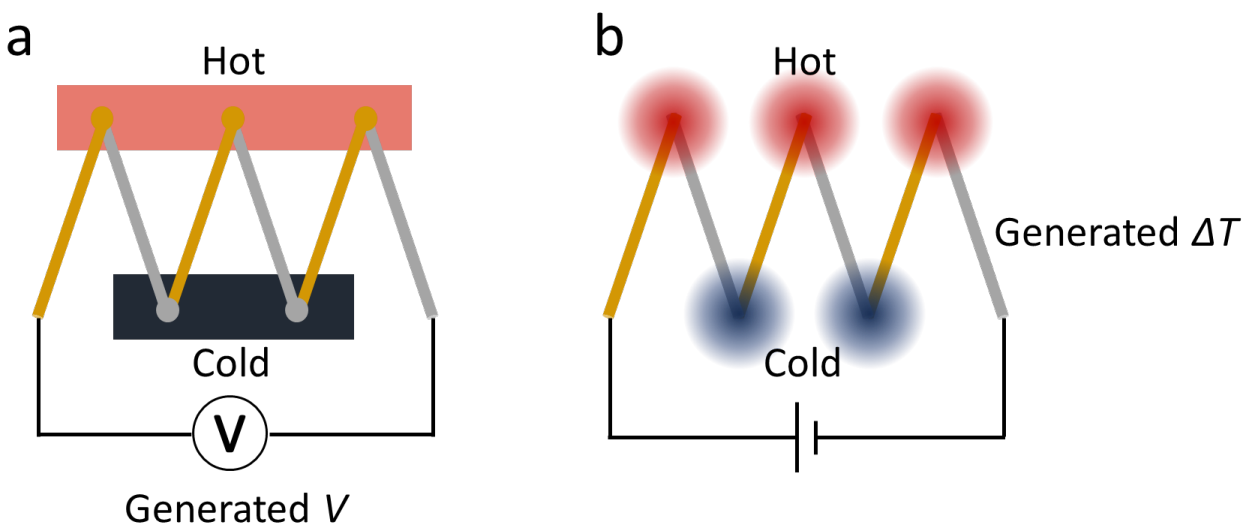

Figure 3.1 Schematic illustration of (a) Seebeck effect, (b) Peltier effect.

The interplays between electrical current and heat current can be expressed by the thermodynamic relationships:

$$
\begin{aligned}
& J=\sigma\left(\frac{d V}{d x}-\alpha \frac{d T}{d x}\right) \\
& Q=-\kappa \frac{d T}{d x}+\alpha T J
\end{aligned}
$$

where $J$ is the electric current density, $Q$ is the heat current density, $\sigma$ is the electric conductivity, $\alpha$ is the Seebeck coefficient, and $\kappa$ is the thermal conductivity at zero electric field condition. Metals generally have high electrical conductivities but low Seebeck coefficients, while insulators have high Seebeck coefficients but low electrical conductivities. Therefore, in real applications, thermoelectric materials are typically semiconductors or semimetals, which possess reasonable values of electrical conductivity and Seebeck coefficient. Beside electrical conductivity and Seebeck coefficient, thermal conductivity is another critical parameter for a thermoelectric material, as such a material should maintain a high temperature gradient to keep a high thermo-induced voltage. Hence, the thermoelectric figure-of-merit (ZT) used to describe the property of a thermoelectric material is a combination of those three materials properties: Seebeck coefficient, electrical and thermal conductivities. 


$$
Z T=\left(\frac{\sigma \alpha^{2}}{\kappa}\right) T
$$

A good thermoelectric material is supposed to have a high value of ZT, which generally requires a high electrical conductivity, a high Seebeck coefficient and a low thermal conductivity. ZT depends also on the temperature since all those properties depends on the temperature. Hence, there are thermoelectric materials working in high temperature range and other types of materials operating best in low temperature range. Good values of ZT are typically above 1. Many good thermoelectric materials are composed of rare atomic elements; which has triggered the search for new classes of materials such as silicides, metal oxides, and metal sulfides. For low temperature application $\left(\mathrm{T}<200{ }^{\circ} \mathrm{C}\right)$, there are no good thermoelectric materials based on abundant atomic elements and the best material is based on bismuth-telluride alloys. This was one motivation why researchers started to investigate if conducting polymers could be potential thermoelectric materials.

\subsubsection{Electrical conductivity}

Electrical conductivity describes the material's ability to transport charge carriers with a given electrical potential difference. The conductivity of a material can be measured and calculated as shown in Figure 3.2.

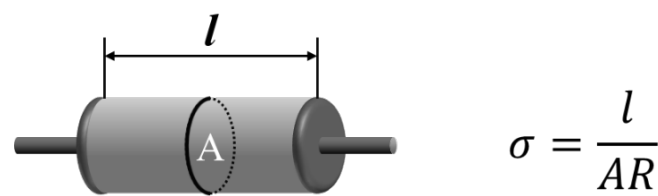

Figure 3.2 Conductivity $(\sigma)$ measurement setup and the calculation equation, where $R$ is the resistance, $l$ is the length, and $A$ is the cross-sectional area of the material.

The unit of conductivity is Siemens per meter $\left(\mathrm{S} \mathrm{m}^{-1}\right)$ or Siemens per centimeter $\left(\mathrm{S} \mathrm{cm}^{-}\right.$ ${ }^{1}$ ), where $1 \mathrm{~S}$ equals to $1 \Omega^{-1}$. According to their conductivities at room temperature, materials can be divided into three categories, which are metals $\left(\sigma>10^{2} \mathrm{~S} \mathrm{~cm}^{-1}\right)$, semiconductors $\left(10^{-10}<\sigma<10^{2} \mathrm{~S} \mathrm{~cm}^{-1}\right)$, and insulators $\left(\sigma<10^{-10} \mathrm{~S} \mathrm{~cm}^{-1}\right)$. 

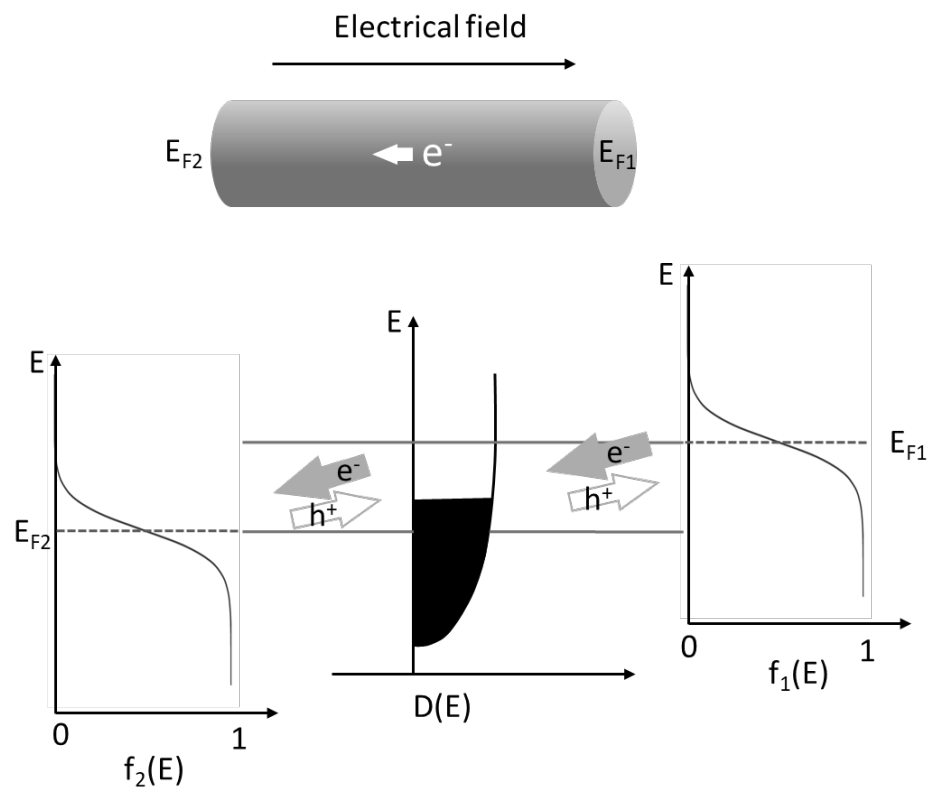

Figure 3.3 Schematic diagram of electrical current generation by applied electrical field.

To generate an electrical current in a material, one method is to apply an electrical field on it, as shown in Figure 3.3. The material has an identical Fermi function between the two ends in the original state. By adding an electrical potential, the Fermi function on the two ends will be shifted along the energy axis. The Fermi function connected to the negative electrode $\left(f_{1}(E)\right)$ is now above the positive electrode side $\left(f_{2}(E)\right)$, and the energy offset is related to the applied voltage:

$$
\Delta E_{F}=E_{F 1}-E_{F 2}=q V
$$

where $q$ is the elemental charge and $V$ is the applied voltage/electrical field. Because the energy levels are not aligned, electrons from the negative electrode will travel down into the empty density of states (DOS) of the material that are lower than the Fermi energy $E_{F 1}$. The DOS is a function representing the number of states that can be occupied at each energy level. In the positive electrode side, meanwhile, the electrons leave the material with Fermi energy $\mathrm{E}_{\mathrm{F} 2}$ and travel in the external circuit. By repeating these processes, the current is generated. The number of states in the range of $d E$ is $D(E) d E$. However, for calculating the current flow, the states only on one of the two 
sides should be counted. We then can calculate the contribution of the current $I(E)$ at the energy level $\mathrm{E}$ as the ratio between the amount of charges $Q(E)$ moving per transit time $t(E) . t(E)$ is the time that one electron of this energy level spends in the material. Since one state can be occupied by one electron, amount of charges at the level $\mathrm{E}$ is $q D(E)\left[f_{1}(E)-f_{2}(E)\right] d E$, where $f(E)$ is Fermi function.

$$
I(E)=\frac{Q(E)}{t(E)}=\frac{q D(E)\left[f_{1}(E)-f_{2}(E)\right] d E}{2 t(E)}
$$

Thus, the current $I$ can be expressed as:

$$
I=\int_{-\infty}^{+\infty} d E \frac{q D(E)\left[f_{1}(E)-f_{2}(E)\right]}{2 t(E)}=\frac{q D\left(E_{F 1}-E_{F 2}\right)}{2 t}
$$

where $D$ is the number of states that is proportional with volume of the material. For a pure ballistic conductor, where electrons are transported in one direction without any diffusion, the transfer time is:

$$
t_{B}=\frac{L}{\bar{v}}
$$

where $L$ is the length of the conductor in transfer direction, and $\bar{v}$ is the average velocity. Hence, for a ballistic conductor, the conductance is:

$$
G_{B}=\frac{I}{V}=\frac{q^{2} D \bar{v}}{2 L}
$$

And for a general conductor including scattering of electronic carriers during the transport, the conductance should be expressed as:

$$
G=\frac{G_{B} \lambda}{L+\lambda}
$$

where $\lambda$ is the mean free path, which can be seen as the average length an electron can travel freely before a collision or a scattering event. It can be also expressed from the diffusion coefficient $\bar{D}$ and average velocity $\bar{v}$ :

$$
\lambda=\frac{2 \bar{D}}{\bar{v}}
$$

Hence, the conductivity of such a conductor can be generally expressed as: 


$$
\sigma=\frac{G L}{A}=\frac{\frac{G_{B} \lambda}{L+\lambda} L}{A}
$$

where $A$ is the cross-sectional area. For a long conductor, where $L \gg \lambda$, this equation can be simplified as:

$$
\sigma=\frac{G_{B} \lambda}{A}
$$

According to Einstein relation, electrical mobility is:

$$
\mu=\frac{q \bar{D}}{k T}
$$

where $\mu$ is the electrical mobility and $k$ is the Boltzmann constant.

Then conductivity can be expressed as:

$$
\sigma=\frac{G_{B} \lambda}{A}=\frac{q^{2} D \bar{D}}{L A}=q\left(\frac{q \bar{D}}{k T}\right)\left(\frac{D k T}{L A}\right)=q \mu n
$$

where $n$ is the charge carrier density. This equation shows that the conductivity of a material is related to the density and mobility of charge carriers. Because of the FermiDirac distribution, at a non-zero temperature, there exists a certain number of electrons that are thermally excited in higher energy level above $\mathrm{E}_{\mathrm{F}}$, similarly there exist holes as absence of electrons below $\mathrm{E}_{\mathrm{F}}$. As a result, both holes and electrons can be transported in an electric field and in opposite directions because of their opposite charge. As a consequence, we can express the electrical conductivity as:

$$
\sigma=e\left(n \mu_{e}+p \mu_{h}\right)
$$

where $e$ is the unitary charge with a unit of Coulomb (C), $n$ and $p$ are densities of electrons and holes, $\mu_{e}$ and $\mu_{h}$ are the mobility of these charge carriers. Therefore, the conductivity of a material can be improved by increasing the density and mobility of its charge carriers.

For metals, the charge carrier density increases with temperature. Meanwhile, however, the phonon vibration is also improved and more collisions will occur between electrons and nucleus. Hence, the resulting macroscopic conductivity decreases with an increased temperature. 
For a semiconductor, there is a band gap between valence band and conduction band. Electrons must be promoted across the band gap into the conduction band to be transported and give rise to an electrical current. If the gap energy $\left(\mathrm{E}_{\mathrm{g}}\right)$ is small and the temperature is non-negligible, the tail of the Fermi distribution reaches the conduction band meaning that the electrons are thermally excited into the conduction band. The density of these electrons increases exponentially with temperature, so the conductivity is enhanced with temperature. In organic semiconductors, however, the $\mathrm{E}_{\mathrm{g}}$ is large, so that the increase of charge density is negligible with the temperature. However, it is generally observed that the conductivity of disordered organic material increases with temperature, and this can be explained by the increased charge carrier mobility. Indeed, the rate of hopping of charge carriers from one site to the next available site in an organic semiconductor is activated by the temperature. In rare cases, for highly ordered and highly doped organic materials, the conductivity can decrease or have no effect with temperature, and the mechanism is similar with metals.

\subsubsection{Seebeck coefficient}

From non-equilibrium thermodynamics, an electrical current can be generated by thermodynamic forces that can be either an electrical field (electric potential gradient) or a thermal field (temperature gradient). In an open circuit, a thermal field leads to an electric voltage, the open circuit voltage. The Seebeck coefficient describes the property of a material to generate an open circuit voltage when submitted to a given temperature gradient:

$$
\alpha=\frac{V_{o c}}{\Delta T}
$$

where $V_{o c}$ is the measured electrical potential difference of two points of a material, and $\Delta T$ is the temperature difference of these two points. The unit of the Seebeck coefficient is volts per kelvin $(\mathrm{V} / \mathrm{K})$ or microvolts per kelvin $(\mu \mathrm{V} / \mathrm{K})$ as most of the thermoelectric materials have small $\alpha$. In a thermoelectric material, electrons and holes tend to move along the material in the direction of temperature gradient when it is subjected in a temperature gradient, and the mechanism can be approximately expressed in Figure 3.4. 

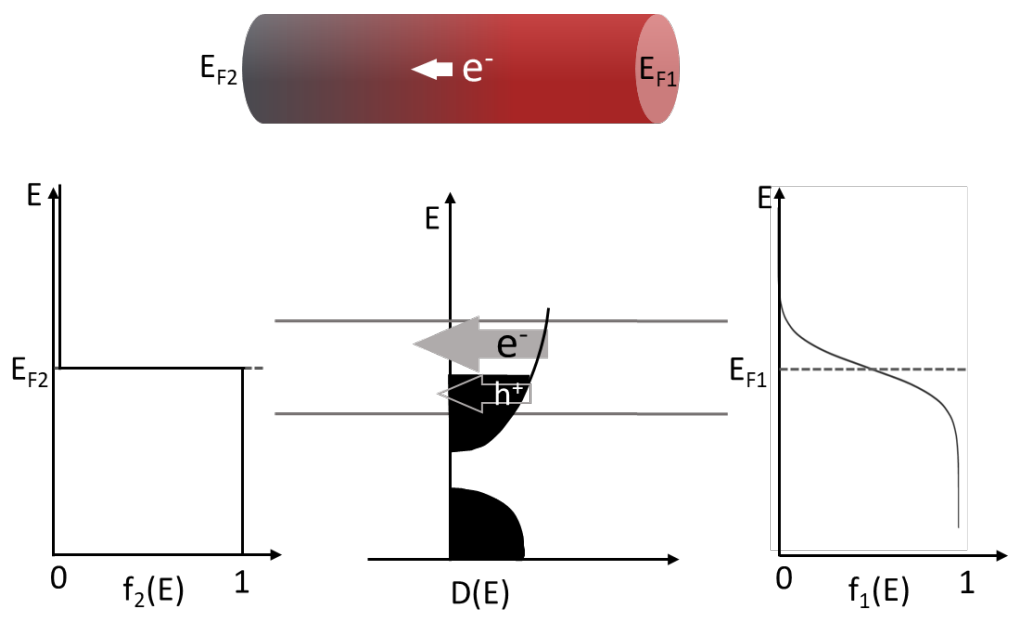

Figure 3.4 Schematic diagram of electrical potential difference generated by applied temperature gradient. Here we assume the temperature of cold side $\left(\mathrm{f}_{2}(\mathrm{E})\right)$ is $0 \mathrm{~K}$.

Assume that the temperature of cold contact is $0 \mathrm{~K}$, then the Fermi function changes abruptly from 0 to 1 at the Fermi level, while the Fermi function of the hot contact changes gradually around the Fermi level. Therefore, the electrons with energy above the Fermi level thermodiffuse from the hot side to the cold side, and the electrons with energy below the Fermi level move from the cold side to the hot side (or the holes thermodiffuse from the hot to the cold side). To simplify the explanation, we assume the mobility of holes and electrons to be the same. Since the density of states (DOS) above and below Fermi level are not symmetric, the thermodiffusion currents in the two directions are different in intensity and there exists a resulting net charges at one side. In the case shown in Figure 3.4, where the DOS increases with the energy around the Fermi level, then the dominating electronic current leads to an excess of electron at the cold side and this type of thermoelectric material is called n-type material with a negative Seebeck coefficient. If the DOS around the Fermi level decreases with energy, the current flows will be opposite, and the materials are called p-type materials with positive Seebeck coefficients. Therefore, in a first approximation, the Seebeck coefficient is related to the slope of the DOS at the fermi level. If the DOS does not change with energy at the Fermi level, which is the case of a superconductor, the Seebeck coefficient is zero. If the DOS changes very fast with energy at the Fermi 
level, that is, the slope of DOS at Fermi level is very large, the material has a high Seebeck coefficient. To increase the Seebeck coefficient, therefore, one can change either the DOS or Fermi level. For a highly doped p-type semiconductor, dedoping is a strategy to decrease the Fermi level to a lower location where the slope of DOS is higher, and thus the Seebeck coefficient is increased. That is, the Seebeck coefficient decreases as the doping level increase, which is a reverse behavior with the change of conductivity ${ }^{4}$. The DOS can be changed, for instance, by managing defects or controlling the electronic structure of the materials by material or molecular design through chemical synthesis. A material with high degree of crystallinity possesses a sharp DOS and hence the Seebeck coefficient is high ${ }^{31}$. Thermoelectric behavior of PEDOT system has also been found to be relative to the molecular weight. High molecular weight (long chain) PEDOT has both higher electrical conductivity and Seebeck coefficient. Therefore, the thermoelectric properties of PEDOT can be simultaneously improved by controlling the synthesis ${ }^{50,51}$.

\subsubsection{Thermal conductivity}

Thermal conductivity $\kappa$ describes the material's ability for conducting heat with unit of watts per meter-Kelvin $\left(\mathrm{W} \mathrm{m}^{-1} \mathrm{~K}^{-1}\right)^{52,53}$. Figure 3.5 shows how to define the thermal conductivity of a material, where $Q$ is the heat flux, meaning the heat flow rate per unit area. The heat transport in a material is described by the Fourier law:

$$
\frac{\Delta Q}{\Delta t A}=-\kappa \frac{\Delta T}{\Delta x}
$$

where the left side represents the power per unit area transported and the right side is the thermal conductivity and temperature gradient.

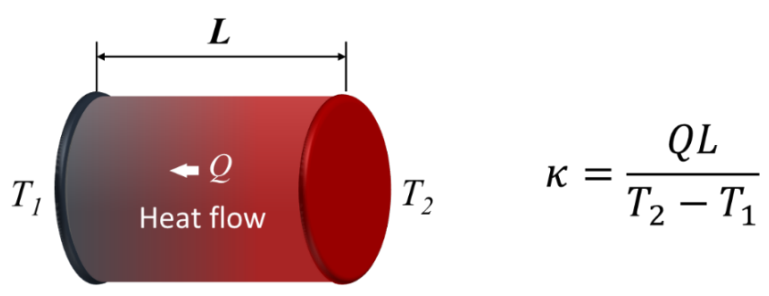

Figure 3.5 Thermal conductivity $(\kappa)$ measurement setup and the calculation equation. 
One of the modes of transport for heat in a material is through phonons, which are quanta of vibrational energy involving the collective motions of many atoms in a solid. The mean free path of the phonon is determined by the morphology of the material. In crystalline materials, the mean free path is large, but it decreases in presence of boundary between crystalline and amorphous regions because of scattering phenomenon. At a given temperature, there will be a certain equilibrium distribution of phonons in a material. With a temperature gradient, the phonons distribution will be disturbed. Meanwhile, phonon-phonon collisions occur in the system to restore the equilibrium distribution and the heat then be transferred by this process. However, phonons can be scattered by other phonons, defects (impurities, dislocations), electrons, and boundaries. These scattering events slow down the transport of phonons and cause the thermal resistance. Thermal conductivities of diamonds are high because of their near-perfect crystallinity and purity. In contrast, polymers generally have much lower crystallinity and purity; and therefore have more boundaries and defects. Hence the thermal conductivities of polymers $\left(0.01-2 \mathrm{~W} \mathrm{~m}^{-1} \mathrm{~K}^{-1}\right)$ generally much lower than ceramics because of the heavy phonon scattering ${ }^{54}$. In most amorphous insulating materials, the thermal conductivity values are rather similar and close to the minimum thermal conductivity formula given by the simple kinetic formula ${ }^{55}$ :

$$
\kappa_{p}=\frac{1}{3} C v L
$$

where $L$, the phonon mean free path, is taken as the smallest distance in the solid: interatomic distance or interchain distance for polymer. $v$ is the velocity of sound and $C$ is the classical specific heat.

The other mode of transport for heat is through electronic charger carriers. Free electrons carry most of the heat from one atom to the others in metals; therefore, metals with a high electrical conductivity generally have a high thermal conductivity. The electronic thermal conductivity is generally expressed by the Wiedemann-Franz law:

$$
\kappa_{e}=T \sigma \mathcal{L}
$$

where $\kappa_{e}$ is the electronic thermal conductivity, $\mathcal{L}$ is the Lorenz number. $\mathcal{L} \approx 2\left(\frac{k_{B}}{q}\right)^{2}$ for non-degenerate materials, such as undoped semiconductors. And $\mathcal{L} \approx \frac{\pi^{2}}{3}\left(\frac{k_{B}}{q}\right)^{2}$ for 
fully degenerate materials like metals and highly doped semiconductors. Approximately, the value of $\kappa_{e}$ is depending on the electrical conductivity.

Hence, the thermal conductivity of a material can be generally expressed as:

$$
\kappa=\kappa_{e}+\kappa_{p}
$$

where $\kappa_{e}$ is the electronic thermal conductivity; and $\kappa_{p}$ is the phonon thermal conductivity. For doped semiconductors, i.e. conducting polymers, it is important to consider both contributions. For low conductivity materials such as ceramics and polymers, $\kappa_{p} \gg \kappa_{e}$, almost no free electrons carry heat. Therefore, the thermal conductivities for such materials is dominated by $\kappa_{p}$.

\subsection{Charge transport}

The electrical current passing through a material submitted to an electrical field or a thermal field is the macroscopic quantity averaging over many events related to the microscopic transport of the electronic or ionic charger carriers.

\subsubsection{Electronic transport}

The electrical conductivity of a material depends on the density and mobility of the charge carriers in the material. For conducting polymers such as PEDOT, the charge carrier density can be enhanced by either chemical doping, or electrochemical doping through redox processes that involve an electron transfer, respectively from a chemical or from an electrode. In conjugated polymers or molecules, the excess electronic charges (electron or hole, which is a lack of electron) will occupy $\pi$-molecular orbitals (LUMO for an excess electron and HOMO for a hole). Because those $\pi$-molecular orbitals are delocalized and the high electron-phonon coupling, the presence of the excess charge in a polymer chain triggers a reorganization of the bond length alternation. Hence, a charged geometrical distortion is formed along the polymer chain, which is called a polaron. If the excess charge is (doubly) positive, it is a positive (bi) polaron; if it is (doubly) negative, it is a negative (bi) polaron.

Neutral PEDOT chain has an aromatic form in the bond alternation as shown in Figure 3.6a. Upon doping, an electron is removed and this triggers a change in the chemical 
structure to increase the quinoid character of the polaron (Figure 3.6b). Because the movement of a positive charge influences its neighboring atoms (6-7 monomer units), one can assume a polaron as a charge carrier unit ${ }^{56,57}$. In the case of low doping level, it is likely that the polaron truly moves along the polymer chains in the electric field, while a high doping level, it is most likely that there is an electron transfer, polaron hopping, from one polaronic site to the next available site.

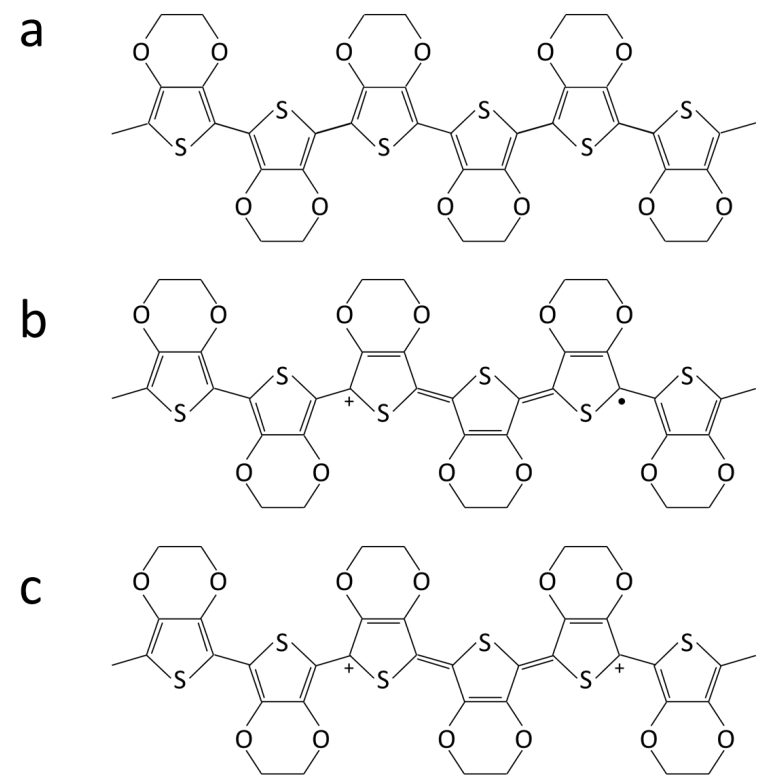

Figure 3.6 Chemical structure of (a) PEDOT neutral chain; (b) PEDOT polaron; (c) PEDOT bipolaron.

Polarons widely exist in lightly doped PEDOT. In highly doped PEDOT chains, two polarons have opportunities to be close to each other and form a bipolaron. There is a progressive change in the bond length alternation from neutral to polaron and bipolaron; such that the aromatic character is replaced by a quinoid character within the charged distortion.

Density functional theory (DFT) has been used to perform quantum chemical calculations of macromolecules and crystallite solids to understand their electronic structure $^{57,58}$. A neutral PEDOT chain with length of 18 monomers shows a band gap 
$\approx 5.33 \mathrm{eV}$ between HOMO and LUMO. Once an electron is removed from the chain, the spin degeneracy is lifted between spin-up and spin-down states, and a new empty energy level (a state) that corresponding to a spin species is generated between HOMO and LUMO, which is called polaron state. If two electrons are taken away from the PEDOT chain, according to Pauli principle, all states remain degenerate and therefore a double degenerated state is generated between HOMO and LUMO, which is called bipolaron state. By removing more electrons from the chain, the spin degeneracy is always lifted if the number of removed electrons is odd; whereas bipolaron states are doubly degenerated. That is, the number of empty electronic states equals to the removed electrons/half of the removed electrons if the number of these electrons is odd/even. For a PEDOT crystallite (3-10 stacked chains), several polaron/bipolaron states interact with each other so that an empty band appears, where the number of states equals to the total number of removed electrons in this crystallite. From a macroscopic viewpoint, e.g. a thin film of PEDOT:PSS, many PEDOT crystallites exist and the band structure of such a thin film is the average of energy spectra of these crystallites $^{59,60}$. The charge carrier density is increased because of the presence of polarons and bipolarons. Therefore, the conductivity of doped PEDOT is significantly higher than neutral PEDOT.

The charge carriers' mobility is a crucial parameter governing the electrical conductivity. The bottleneck for the charge transport (the slowest serial transport phenomenon) in conducing polymers is the interchain (intermolecular) transport. Indeed, the intrachain transport is fast but the lengths of conjugated polymers are generally not large, so that hopping between neighboring chains is demanded for charge transport. An efficient charge transport requires that the charge carries are not trapped or scattered. However, many factors affect the charge carrier mobility in conjugated polymers such as impurities, molecular packing, and disorder. This can explain why the charge mobility of single crystalline silicon $\left(1400 \mathrm{~cm}^{2} \mathrm{~V}^{-1} \mathrm{~s}^{-1}\right.$ for electrons) is much higher than that of conjugated polymers (few tens of $\mathrm{cm}^{2} \mathrm{~V}^{-1} \mathrm{~s}^{-1}$ ) Sirringhaus et al. studied the relationship between regioregularity and charge mobility of poly-3-hexylthiophene (P3HT) ${ }^{62}$. They found that the level of regioregularity affects the molecular ordering in lamella structure obtained by self-organization upon solvent evaporation. Films of P3HT with high regioregularity P3HT (96\%) possess a mobility 
two orders of magnitude higher than those of low regioregularity (81\%). Later it was shown that local molecular order in an overall disordered material could lead to decent charge carrier mobility and that high molecular weight, long polymer chains could promote high mobility ensuring the charge passing from one nanocrystal to the other 63,64

Temperature-dependent electrical conductivity measurement helps to identify the charge transport mechanism of a material. For metals, although the charge carrier density increases with an increased temperature, the conductivity decreases because of decreased transport mobility led by collisions between nuclei and transported electrons. This negative temperature-dependent conductivity is a sign of metallic transport that happens on metals and a few conducting polymers ${ }^{31,65}$. For most semiconductors, there is a positive correlation between temperature and conductivity. However, the mechanism can be different for crystalline and disordered materials. The band gap of some inorganic semiconductor crystals is relatively small; hence, a large number of electrons can be excited from valence band to conduction band. In this case, the increased temperature causes an increased concentration of charge carrier, which further leads to an increased conductivity. For undoped organic semiconductors, the band gap is large and the thermal excitations thus are negligible. For doped and disordered organic semiconductors, for instance, PEDOT:PSS, the band gap is vanishingly small, hence thermal excitations could in principle be possible, but the charge carrier mobility is so low that it is the dominant effect since the slight increase in charge carrier concentration by thermal excitation is negligible. PEDOT:PSS displays a large optical absorption in the infrared range indicative of free electrons in Drude models ${ }^{66}$, thus indicating that some PEDOT-rich nanodomains are metallic-like. On the other hand, the charge carrier mobility is mostly thermally activated indicating that these PEDOT-rich nanodomains are not well electrically connected and that the bottleneck in the transport is between those PEDOT nanodomains.

PSS is an electronic insulating phase in PEDOT:PSS, hence the distribution of PSS in PEDOT:PSS governs its electrical conductivity. PEDOT:PSS has typically an excess amount of PSS ( 2 or 3 times more than PEDOT in PH1000), in order to disperse the nanoparticles of PEDOT:PSS in water. Indeed, the negative chains of the PSS forms 
like a shell around the PEDOT:PSS nanoparticles in the water emulsion which prevent them to aggregate by electrostatic repulsion. It is widely accepted that by adding high boiling point solvent such as dimethyl sulfoxide (DMSO) or ethylene glycol (EG), the non-conductive PSS chains in excess can be phase separated or partially removed from the PEDOT-rich domains, leading a better bridging among these PEDOT nanodomains; the polymer chains themselves were claimed to change from coil structure into expanded-coil or linear structure ${ }^{67-69}$. Secondary doping makes the domains closer to each other, and slightly tighten the $\pi$-stacking, hence the charge transport become easier. In other words, the secondary dopants lead to the growing size of gel-like domains accompanied with purification of PEDOT-rich cores, and therefore the charge mobility is significantly enhanced ${ }^{70-72}$. Hence, we call primary doping a change in oxidation level of PEDOT:PSS and secondary doping a change in morphology in the film. This morphological change increases the electrical conductivity from $0.1 \mathrm{~S} / \mathrm{cm}$ to $1000 \mathrm{~S} / \mathrm{cm}^{73-75}$. Figure 3.7 shows the widely accepted secondary doping mechanism on morphology changing. In summary, chemical or electrochemical doping is the promising way to increase the charge carrier density. And improving crystallinity/regioregularity, decreasing non-conductive impurities and grain boundaries, and reducing the macroscale disorder are the most widely accepted strategies for charge mobility enhancement.

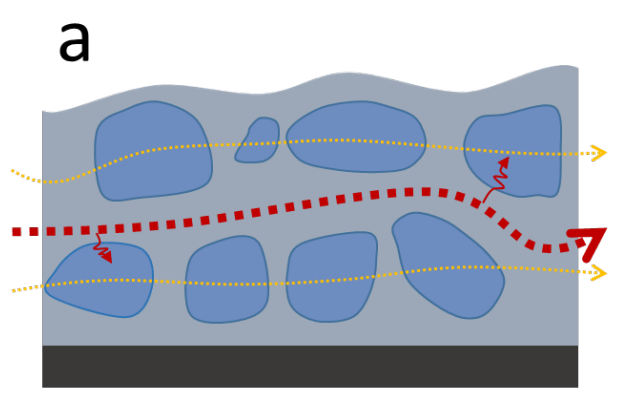

PEDOT-rich domains b

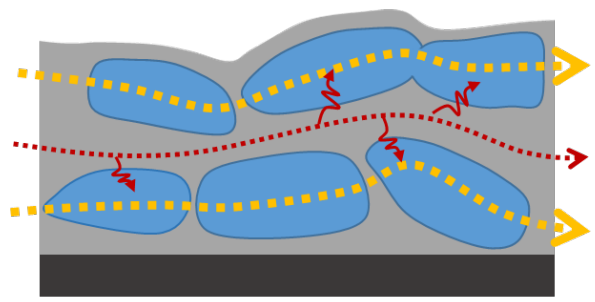

Excess PSS

Figure 3.7 Schematic diagram of PEDOT:PSS before (a) and after (b) secondary doping. The yellow lines show the electronic charge transport routes; the red lines show the ionic charge transport routes ${ }^{72,76}$. 


\subsubsection{Ionic transport}

Beside electronic conductor, PEDOT:PSS is also a good ionic conductor under some appropriate conditions such as in hydrogel state or in high humidity environment ${ }^{77}$. Although the mechanism of ionic transport is not fully understood, it is widely accepted that the hydration of PEDOT:PSS creates ion-transport pathways in the PSSrich phase $\mathrm{e}^{72,76-78}$. Cations like $\mathrm{H}^{+}$or $\mathrm{Na}^{+}$are travelling mainly in the PSS-rich regions, and simultaneously, partially diffusing into the PEDOT-rich domains, as shown in Figure 3.7. Secondary dopant enlarges the PEDOT-rich domains, therefore the diffusion of ionic transport is enhanced in the PSS-rich phase, while the ionic mobility decreased in the PEDOT-rich phase ${ }^{72}$ (Figure 3.7b). For a non-hydrogel PEDOT:PSS sample, such as a film, the ionic conductivity exponentially increasing with the relative humidity $(\mathrm{RH})^{76,79,80}$, because PSS is highly hygroscopic. This is a unique property for PEDOT:PSS compared to PEDOT:Tos which possess a much lower ionic conductivity.

Thermodiffusion of matter, also called the Soret effect, has been known for a long time, which describes the diffusion of molecules/particles along temperature gradient and typically towards the cold side ${ }^{81}$. In an electrolyte, cations and anions thermodiffuse. If their thermodiffusion rate is not equal or not in the same direction, there will be an excess of one type of ions at the cold side, such that an open circuit voltage $\left(V_{o c}\right)$ is generated on two sides of the electrolyte submitted to a temperature gradient. Hence, electrolyte can also be characterized by an ionic Seebeck coefficient. For instance, in a high humidity environment, PEDOT:PSS is able to generate higher $V_{o c}$ than that in low humidity environment under a temperature gradient which has been explained as ionic thermal voltage ${ }^{80,82}$. It has been found that the $V_{o c}$ generated in an electronic conductor (e.g. PEDOT:Tos) follows the time dependent increase with $\Delta T$; thus revealing that the thermodiffusion of electronic charge carrier is rapid. For a pure ionic conductor (e.g. PSSNa), on the contrary, $V_{o c}$ increases much slower than the $\Delta T$, as the mobility of $\mathrm{Na}^{+}$is very low. For an electronic-ionic mixed conductor (e.g. PEDOT:PSS), $V_{o c}$ contributed by both electrons and ions and the time dependence of the Seebeck voltage is peculiar. As a $\Delta T$ is applied, $V_{o c}$ increases, due to the thermodiffusion of cations in PSS, to a maximal value followed by a decrease to a minimal Seebeck coefficient. The minimal thermovoltage obtained at steady state is 
attributed to the electronic Seebeck coefficient and it is identical as for the film exposed at low humidity ${ }^{82}$. As shown in Figure 3.8.

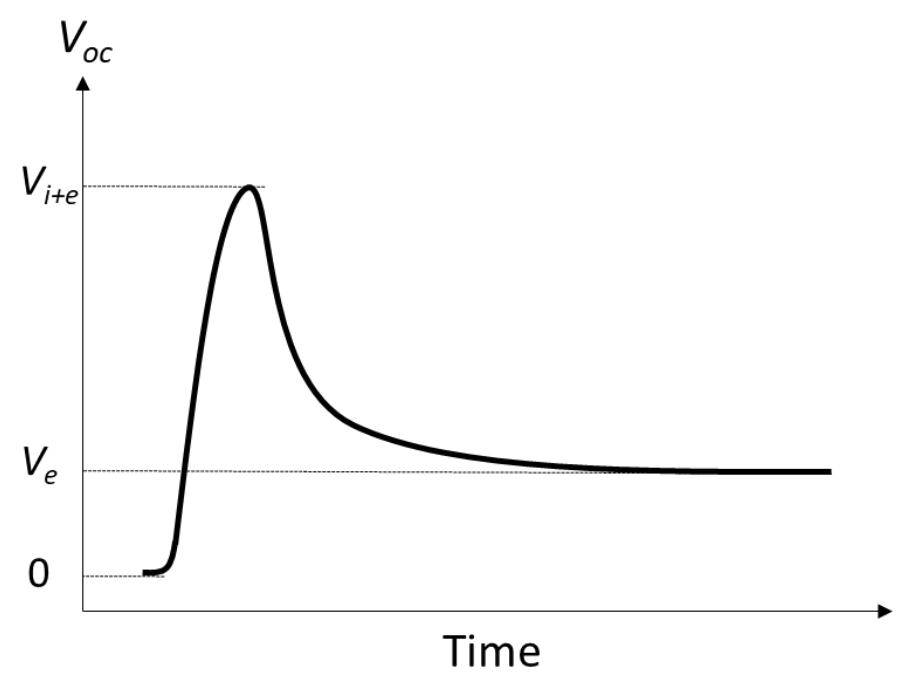

Figure 3.8 Schematic of a typical response of $V_{o c}$ with a $\Delta T$ in high humidity environment (e.g. $\mathrm{RH}=70 \%$ ) of PEDOT:PSS.

In this process, cations accumulate to the cold side and establish an ionic thermovoltage, which is relatively high according to Soret effect. It should be noted that ions cannot pass through the electrodes. Therefore, simultaneously, due to this ionic thermovoltage, the electrons inside the material start reorganizing and the doping level through the material is changing locally. Hence, the ionic thermovoltage decreased. After a while, a new steady state is established between electrons and ions, so that the measured thermovoltage tend to the electronic thermovoltage. Since the ionic thermovoltage is related to $\mathrm{RH}$ and $\Delta T$, with a known $\Delta T$ and a measured ionic thermovoltage, the RH can be then calculated. Although it is not stable with time, the ionic Seebeck effect can be exploited for ionic supercapacitors or sensors ${ }^{83-85}$. 


\subsection{Optical absorption}

Light is electromagnetic radiation that propagates as waves and particles called photons. Solar light that reaches earth has wavelength in the range between about 250 $\mathrm{nm}$ and $2500 \mathrm{~nm}$, as shown in Figure 3.9. Sunlight at sea level has much lower energy than that above the atmosphere, which is due to the absorption of air, water and flying particles. Solar energy has gained a lot of research interest because it is by far the largest renewable energy available. Good absorbers that are employed in two key technologies, solar heating and photovoltaics, are able to absorb large amount of energy in the visible and infrared ranges, where most of the solar energy is contained 86,87 .

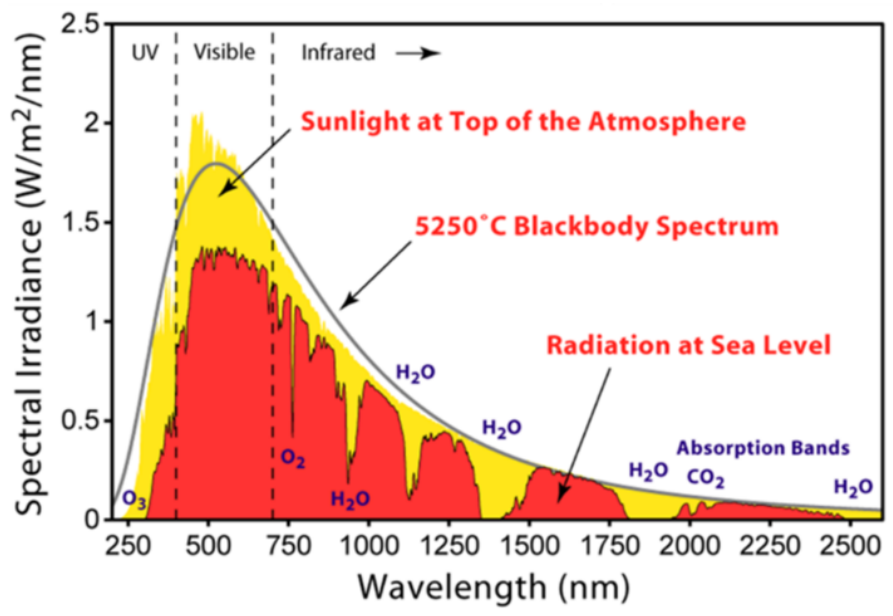

Figure 3.9 Solar radiation spectrum (source: commons.wikimedia.org).

Electrons of atoms/molecules have different accessible quantum energy levels, such that when a photon of a given frequency $v$ strikes a material, the energy of the light hv can be absorbed by the electrons of energy $E_{i}$, if there is an accessible empty energy level $\mathrm{E}_{\mathrm{f}}=\mathrm{E}_{\mathrm{i}}+\mathrm{h} v^{87,88}$. The absorbed light energy can be further transformed to other types of energy such as thermal energy (heat) or electricity.

Absorption spectroscopy is a modern measurement technique that is typically used to determine the presence and concentration of a substance in a sample, by measuring its 
absorption spectrum. Ultraviolet-Visible-Near infrared (UV-Vis-NIR) absorption spectroscopy is one of the most widely used methods for investigating the electronic structure of organic materials such as polymers. There are three types of molecular orbitals that represent the electrons in conjugated polymers; $\sigma$-electrons, $\pi$-electrons, and non-bonding electrons ( $n$-electrons), as shown in Figure 3.10. By absorbing different energy photons (i.e. different wavelengths of radiation), electrons can reach various unoccupied molecular orbitals and display several optical transitions appearing as peaks in the absorption spectrum.
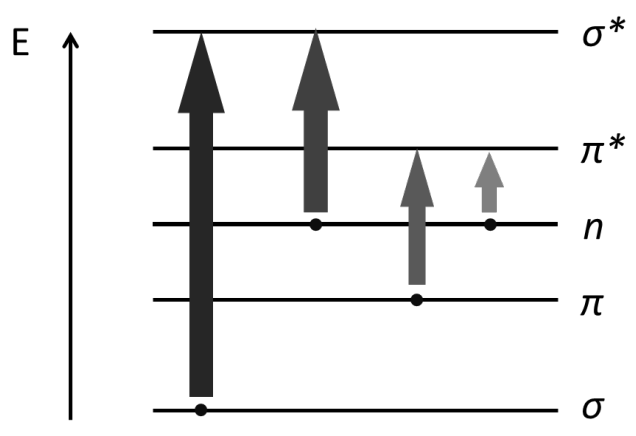

Figure 3.10 Four electronic transition modes, which absorb radiations of different wavelengths.

UV-Vis-NIR absorption spectroscopy has been used to determine the oxidation level of conducting polymers, e.g. PEDOT:PSS thin films ${ }^{57,89,90}$. A typical absorbance spectra of a PEDOT:PSS thin film (oxidation level $=33 \%$ ) has low absorption in the visible range but and continuously increase in the NIR range towards the IR range as a feature of quasi-free electrons. By dedoping the film, the absorption above $1200 \mathrm{~nm}$ continuously decreases along the reduced oxidation level. Highly de-doped film (neutral PEDOT:PSS) shows a new peak around $600 \mathrm{~nm}$. The state of the art theory that has been used to explain the spectra is time-dependent density functional theory $(\text { TD DFT) })^{57}$. PEDOT in its high oxidation level (e.g. pristine PEDOT:PSS) absorbs low energy radiation (about $900 \mathrm{~nm}$ and 1300-1700nm); which corresponds to the excitation of the electrons from the valence band to the polaronic and bipolaronic states. The absorption around $600 \mathrm{~nm}$ corresponds to the transition from valence band to conduction band in neutral PEDOT:PSS segment of polymer chains (undoped). 
Because of this absorption difference along oxidation level, the color of PEDOT:PSS films varies in a range from dark blue to almost transparent, which gives PEDOT:PSS the property of electrochromism since its colors changes upon the application of an electrochemical bias.

\subsection{Water evaporation}

Evaporation is the process of phase transition from a liquid state to a gas state in the form of a vapor. In the water cycle on earth, after evaporation of water from the salted seawater, water condenses in clouds to be back in its liquid form in the form of tiny droplets and then rain falling on the surface of the earth as soft water. Water molecules are strongly polar molecules with dipole moment of 1.8564 Debye resulting in a liquid of high static dielectric constant $(\varepsilon=80)$. Moreover, the electron lone pairs on the oxygen atom and the lack of electron density on the hydrogen atoms leads to the formation of hydrogen bonds between water molecules, which explains the relatively high boiling point for this small molecule liquid and high heat capacity $(4.2 \mathrm{~J} /(\mathrm{g} \cdot \mathrm{K})$ at $\left.25^{\circ} \mathrm{C}\right)$, and high heat of vaporization $\left(2257 \mathrm{~kJ} / \mathrm{kg}\right.$ at $\left.100^{\circ} \mathrm{C}\right)$. By absorbing energy (endothermic process), the hydrogen bonds can be broken and thus the water molecules are released in the gas phase. Once a water molecule in air touches a liquid water surface, it forms new hydrogen bonds with neighboring liquid molecules and energy is released (exothermic process) in the phenomenon called condensation. Evaporation and condensation are happening all the time at the water-air interface through a dynamic equilibrium that depends on temperature and pressure.

Evaporation requires energy from the surroundings and can lead to a cooling effect (endothermic process). The evaporation rate can be significantly increased by applying extra energy such as solar radiation. One strategy is using concentrated solar light to heat up high heat capacity fluids (e.g. synthetic oil, molten salt) and transfer the thermal energy to water. Another method is to illuminate water directly with solar light, which normally needs an extra solar absorber because water molecules only absorb a limited solar energy. Indeed, water absorbs in the UV, while a lot of light from the sun is in the visible and infrared ranges. These solar absorbers can be made by carbon materials ${ }^{26,91}$, metallic nanoparticles ${ }^{92,93}$, and composite materials ${ }^{94}$. 


\subsection{Freeze-drying}

While evaporation describes the phase transition from liquid to vapor, there is also another phenomenon when water molecules undergo a transition from solid phase to vapor phase, which is called sublimation. It occurs at temperatures and pressures below a substance's triple point in its phase diagram, which corresponds to the lowest pressure at which a substance exists in the liquid (Figure 3.11). Freeze-drying is a dehydration process by sublimation under low temperature and low pressure. Freezedrying is used abundantly in various applications, for instance in the food industry.

The case of water removal in a hydrogel is illustrative to explain the feature of freezedrying. A hydrogel is a blend composite made of water and solid polymer, where the mechanical feature of the hydrogel is due to the solid network in which water is imprisoned. During the evaporation of water for a hydrogel, the water evaporates step by step and the polymer particles collapse on each other to create a dry film. In other words, in a normal evaporative drying process, that is, the water evaporates from a wet sample in atmosphere, the solid phase in the sample tends to agglomerate because of the hydrogen bond or Van der Waals force between water molecule and the solid phase, as the water content is decreasing. In contrast, during the freeze-drying of a hydrogel, the solid polymeric content remains at its original position in the gel, while the water is removed, giving a porous structure. Therefore, freeze-drying is one of the key techniques to fabricate aerogels and other porous structures. We have focused on cellulose aerogels created by freeze-drying a hydrogel made of nanofibers of cellulose and water. Mechanical integrity of cellulose aerogel in presence of humidity or water is an important factor that needs to be considered, as cellulose products are generally easily disintegrated when exposed to water because of the hydrogen bond between cellulose chain and water molecule. Therefore, cellulose aerogels typically collapse when dip into water. However, it has been demonstrated that crosslinking is an efficient process to increase the mechanical property. This can be achieved by the addition of crosslinking agents ${ }^{19}$, cyclic freeze-thaw ${ }^{95}$, and freezing-induced crosslinking ${ }^{96}$. Beside mechanical property, the porosity and dimension of the pores are also important features for many applications ${ }^{97,98}$. Porosity and pore dimension can be changed by controlling the content of solid phase in the original hydrogel, as well 
as the freezing rate. Extremely high freezing rate can be achieved, for instance, with the spray freeze-drying technique, resulting in the possibility to have small pore dimensions ${ }^{98}$.

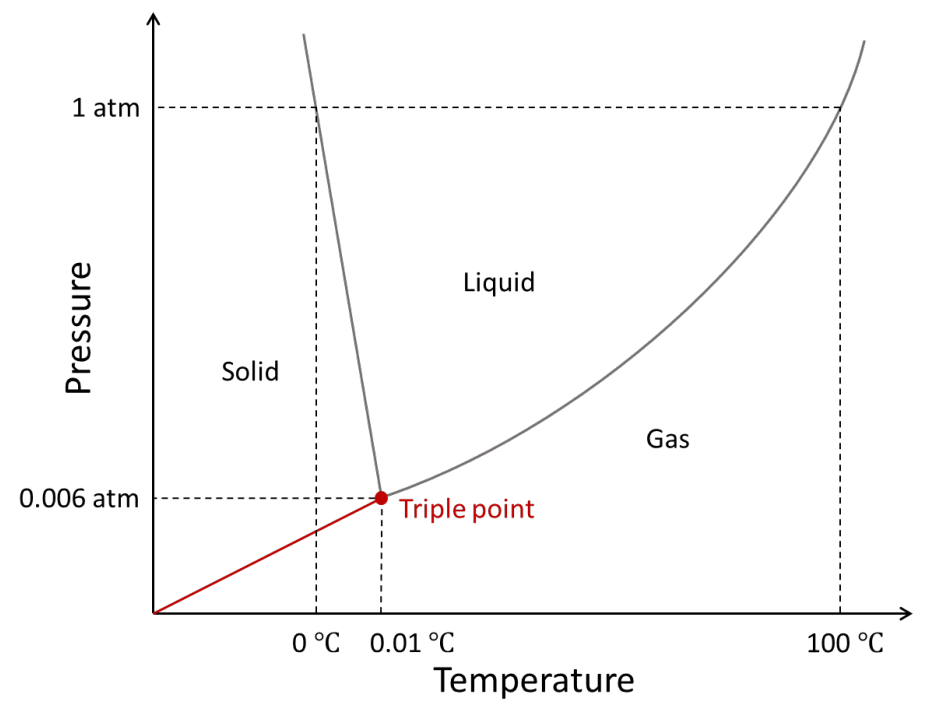

Figure 3.11 Water phase diagram. 


\section{4}

\section{Characterization methods}

\subsection{Electrical properties}

\subsubsection{Conductivity of thin films}

Thin films of the blend PEDOT:PSS with the crosslinking agent (3glycidyloxypropyl)trimethoxysilane (GOPS) were spin-coated on glass substrates with pre-patterned gold electrodes. The resistance of these films was measured by a standard four probe setup as shown in Figure 4.1. ${ }^{99}$

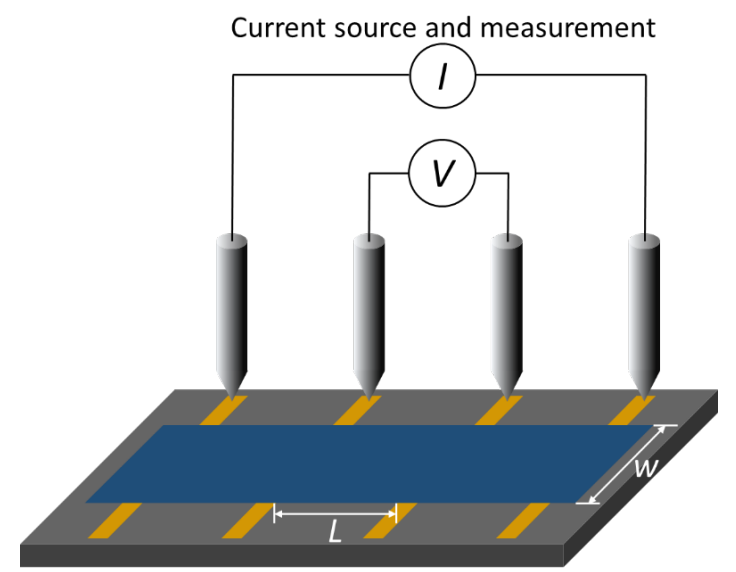

Figure 4.1 Four-point-probe measurement.

A common four-point-probe setup consists of four equally spaced tungsten metal tips. The two outer probes are used to supply the film a high impedance current while the 
two inner probes are connected to a voltmeter that measures the voltage across them. Because of the high impedance of the voltage measurement device, the current flow that passes through the inner probes is negligible. The advantage of this technique over two probes measurement is that the contact resistance is not polluting the measurement. Indeed, in a two-probe measurement, the resistance measured is the equivalent resistance of two resistance in series: the contact resistance and the resistance of the material to be measured. If the material is highly resistive, then the contact resistance might be relative small and can be neglected. But if the material is a good electrical conductor, its resistance can be so small that the contact resistance is dominating.

The film thickness $(t)$ was measured by a Dektak surface profiler. The conductivity can be calculated by:

$$
\sigma=\frac{L}{R w t}
$$

where $R$ is the resistance, which is the ratio of measured voltage and applied current, $L$ the length, and $w$ the width of the corresponding film, as shown in Figure 4.1.

\subsubsection{Resistance and Seebeck coefficient of aerogels}

To characterize the electrical properties of an aerogel, it is not straightforward to use the 4-point probe setup. Instead, we instead measure the resistance of the aerogel in a two-probe setup. For that purpose, it is essential to decrease the contact resistance and measure on a large-size sample to reduce the influence from contact resistance. We managed to make good electrical contacts between the PEDOT/cellulose aerogel and a thin film of PEDOT:PSS used as electrode. Indeed, PEDOT:PSS electrode coated on PMMA has the advantage that it has a good electrical conductivity $(1000 \mathrm{~S} / \mathrm{cm})$ and it is not covered by any insulating oxide layer; which are two prerequisites to provide good electrical contacts. The two-probe resistance is then measured upon application of a pressure and later used as a pressure sensor (Figure 4.2). 


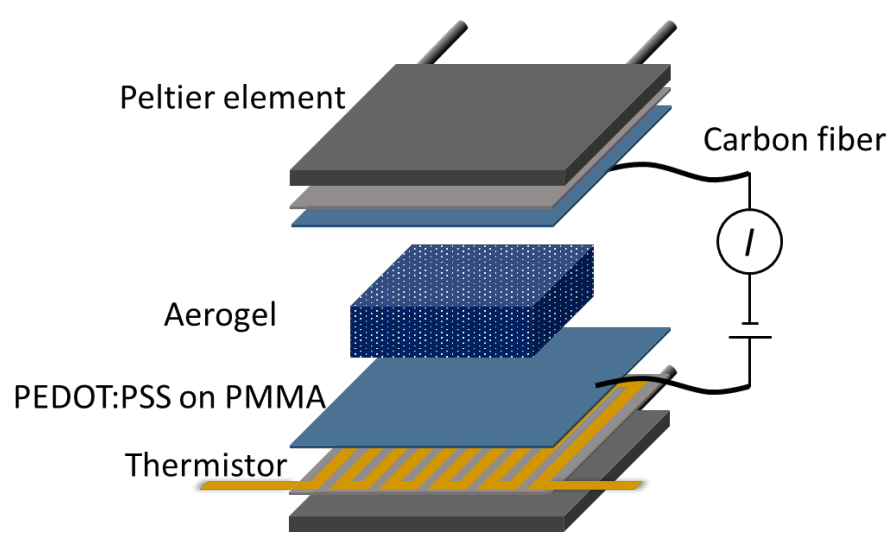

Figure 4.2 Schematic of the setup for measuring resistance and Seebeck coefficient of aerogels.

The setup was used to measure resistance in the same direction as the applied pressure. Two Peltier elements were used under and above the setup to control temperature difference between the two PEDOT:PSS electrode. Temperature gradient can be applied on the two sides of the aerogel, so that the thermovoltage is measured through the PEDOT:PSS electrode. The real-time temperatures at the top and bottom PEDOT:PSS electrode were measured by the calibrated Au-thermistors. The PMMA substrate is so thin that the temperature drop between the thermistor and the electrode is small. These two Peltier elements were connected with a fully automatic LabView controlled program, which provides a temperature gradient at a set time. The PEDOT:PSS films which act as two probes were connected with a Keithley 4200 electronic parameter analyzer. This analyzer provided a changing voltage and recorded the corresponding current; hence, we got current-voltage curves that can be used to calculate the resistance. And the intercept of these curves on voltage axis corresponds to the generated thermo-voltage $\Delta \mathrm{V}=\mathrm{S} \cdot \Delta \mathrm{T}(\mathrm{S}=$ Seebeck coefficient) (See Figure 4.3). 


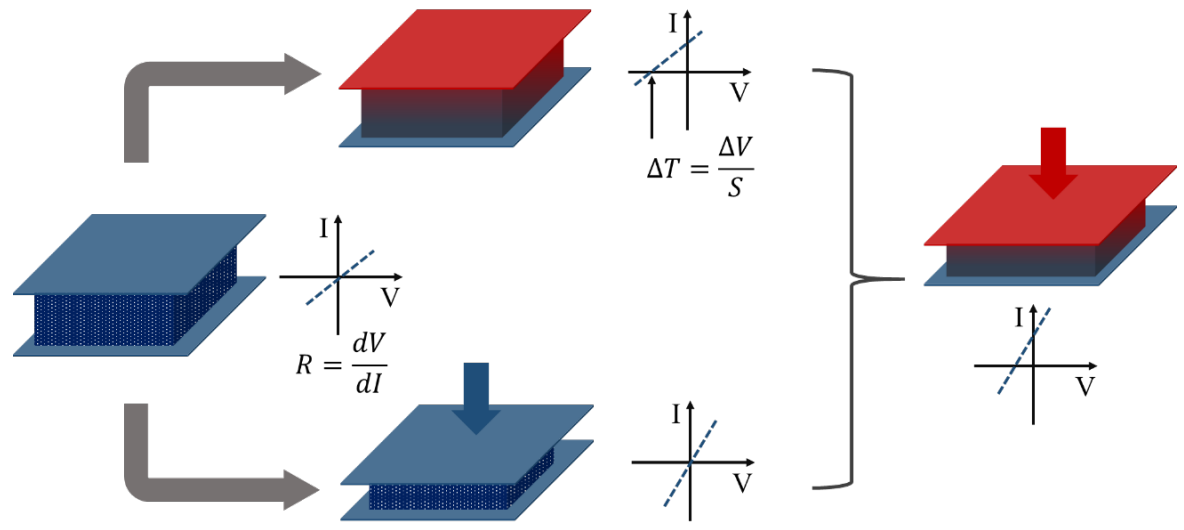

Figure 4.3 Schematic of the concept design of sensing temperature, pressure, and both.

\subsection{Scanning Electron Microscope (SEM)}

SEM is one of the electron microscopes that uses a focused electron beam to scan over the surface of a specimen to create an image. The interactions between the electrons and the atoms of the specimen produce various information about the surface topography and composition of the specimen. A schematic of SEM is shown in Figure 4.4. Electrons are produced from the electron gun on top of a chamber and then be attracted by the anode (positively charged) below the electron gun. There is a hole in the middle of the anode which allows a fraction of electrons pass through it. The electrons are then be focused by a composition of magnetic lens. An objective lens under the condenser lenses is used to scan the beam. The focused electron beam hits the surface of the specimen and mainly two types of electrons come out of the specimen and detected by detectors. Some of the electrons from the beam bounce back out of the specimen which called backscattered electrons are detected by the backscatter electron detector. On the surface of the specimen, atoms absorb energy from the beam and some of the electrons of these atoms come out of the sample, which called secondary electrons. The secondary electrons are then detected and the detector uses the information from these electrons to generate the image on a computer. 


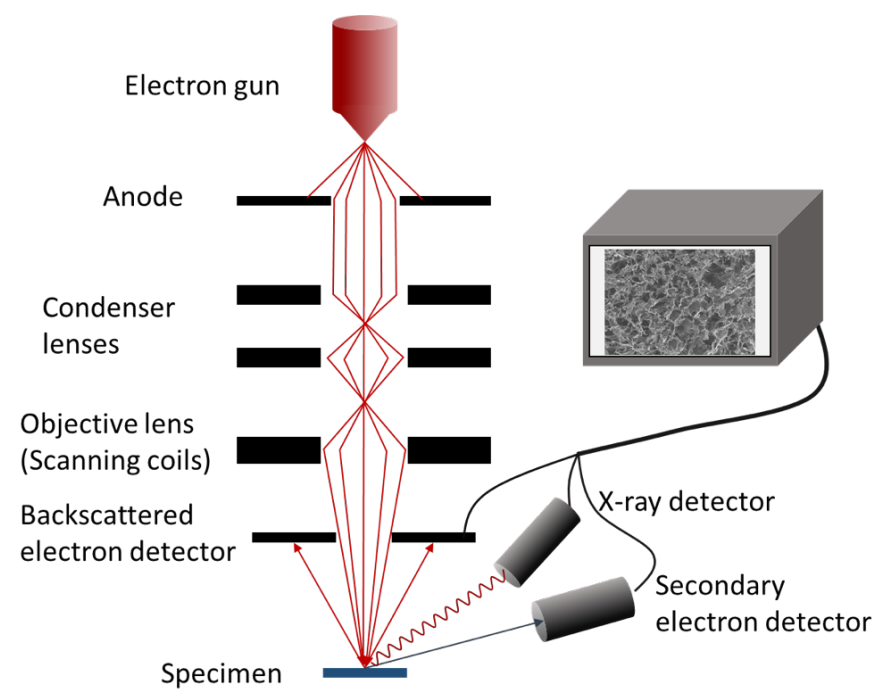

Figure 4.4 Schematic of scanning electron microscope.

In order to investigate the surface morphology and porous structure of PEDOT:PSSGOPS-NFC aerogels, optical microscope and SEM were used. Typical images are shown in Figure 4.5. The aerogels possess open porous structure with dimension from 10 to 100 micrometers depending on the formulation of the hydrogel and the details of the freeze-drying process (cooling rate, thickness, pumping power). For instance, if the starting hydrogel has a low water content, and that a fast freezing rate is used, the resulting pores are small.
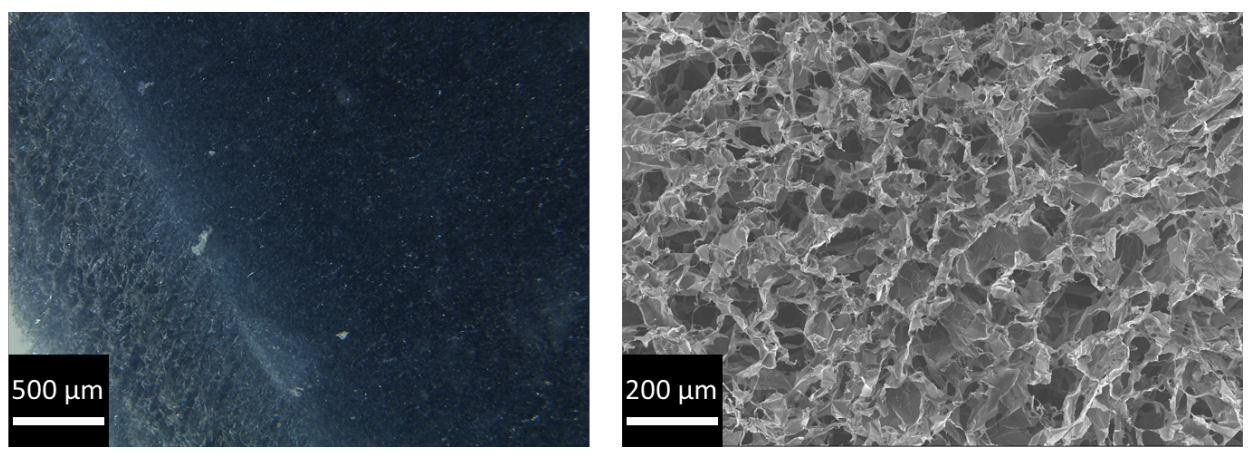

Figure 4.5 Optical (left) and SEM (right) images of PEDOT:PSS-GOPS-NFC aerogels. 


\subsection{Optical absorption}

UV-Vis-NIR absorption spectroscopy is widely used to investigate the optical absorption of a material but also several other properties, such as film thickness, molecular ordering, doped species (redox state), and photo induced charge transfer. Here, a specific interest is the possibility to identify different doping states of the material since neutral polymer chains and polaron/bipolaron absorb different wavelengths of radiation ${ }^{90,100}$. A schematic of UV-Vis-NIR spectrophotometer is shown in Figure 4.6. A beam of incident source light passes into a monochromator through an entrance slit and is dispersed with a diffraction grating. By controlling the angle of the grating, radiation with a specific wavelength exits the monochromator and is directed to a sample and a reference channel. The transmitted radiation of the sample and reference are then detected by two detectors and analyzed by a computer.

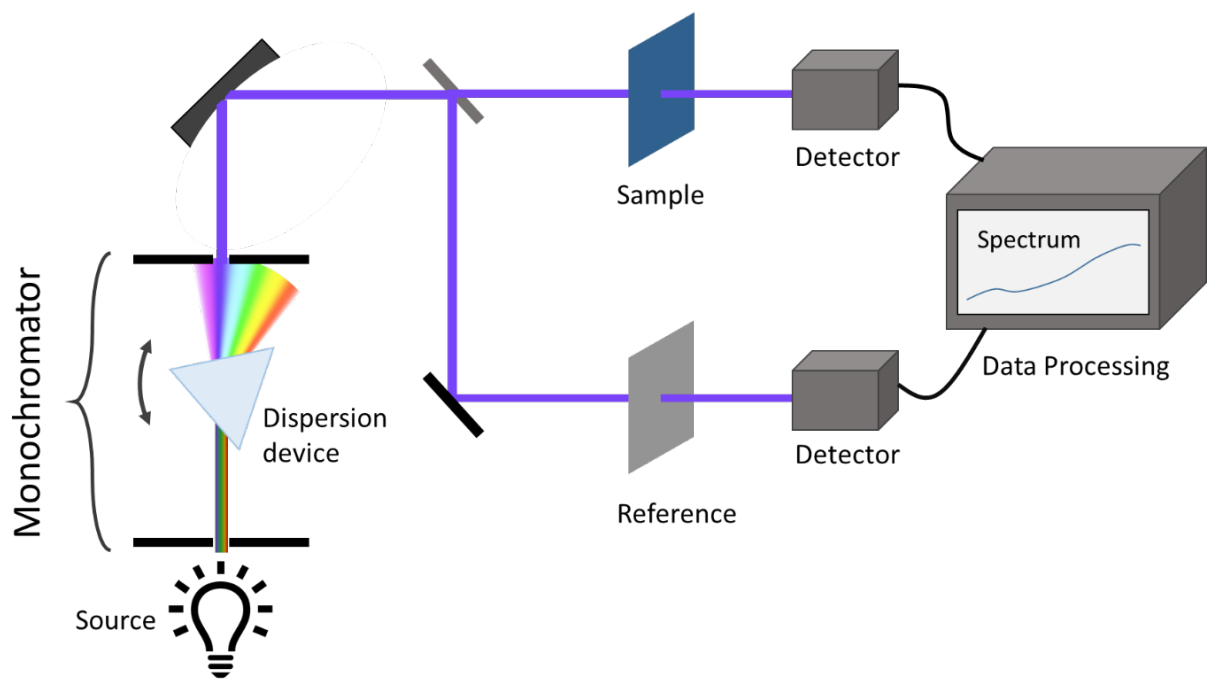

Figure 4.6 Schematic of UV-Vis-NIR spectrophotometer.

The absorbance $(A)$ of a sample can be obtained by:

$$
A=-\log \left(I / I_{0}\right)
$$


where $I$ is the intensity of the radiation that passes through the sample and $I_{0}$ is the incident intensity. The absorbance is reported versus the wavelength to define an optical absorption spectrum. Peaks in that spectrum reveal optical transitions in the material. Figure 4.7 shows an approximate absorption spectrum of poly(3hexylthiophene) (P3HT), that illustrates the optical changes due to doping level. The absorption peak at $520 \mathrm{~nm}$ corresponds to the $\pi-\pi^{*}$ transition of electrons in a neutral P3HT film. Upon doping, polarons and bipolarons are formed, introducing new electronic states with energy levels between the $\pi$ and $\pi^{*}$ states. These sub-gap states can be occupied by electrons absorbing lower energy radiation than required for the $\pi-\pi^{*}$ transition, forming new absorption bands at higher wavelengths ${ }^{101}$.

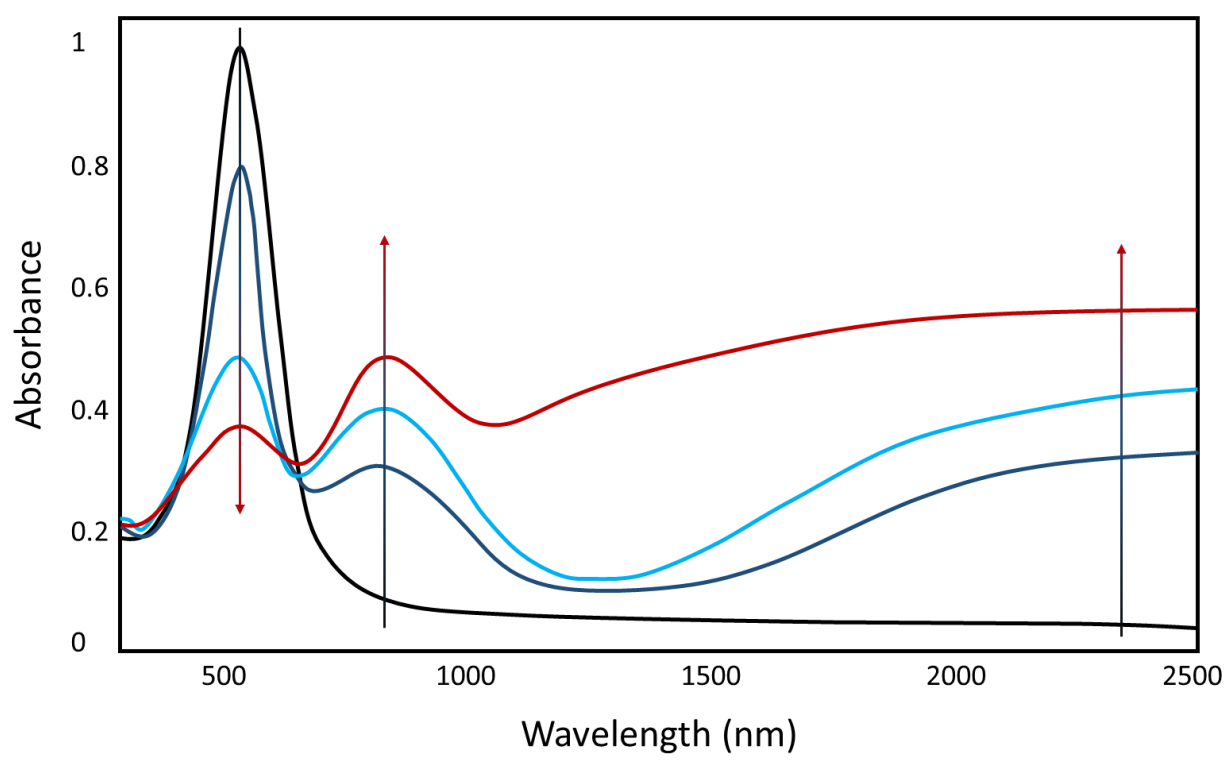

Figure 4.7 Schematic of UV-Vis-NIR absorption spectrum of P3HT film with different doping level (marked by arrows).

In this thesis, UV-Vis-NIR was used in order to understand the effect of added GOPS on the doping level of PEDOT:PSS. We conclude that the charge carrier density is unaffected by crosslinking of GOPS and PSS (see Figure 3 of Paper 1 in the Results section). When light is absorbed by a material, excitons or excited states are created. The fates of those excitons leads to several phenomena: (i) radiation decay by 
fluorescence or phosphorescence when the light is reemitted by the material at a larger wavelength, (ii) the creation of photocurrent through exciton dissociation (used in solar cells), (iii) the quenching of the excitons leading to a non-radiative decay, i.e. heat. In the solar steam generation application (Paper 4), UV-Vis-NIR was used to investigate the absorbance of PEDOT:PSS-GOPS-NFC aerogels in the range of solar light radiation. The use of a conducting polymer enables the absorption in the infrared range of the solar spectra as well as an efficient exciton quenching by the high electronic polarization due to free charge carriers. It has been found that a $2 \mathrm{~mm}$ thick aerogel can absorb $\sim 99 \%$ of the solar light (with $\sim 1 \%$ of reflection and scattering), and this high level of absorbance lead a high light to heat energy conversion efficiency ( $\sim 81 \%)$.

\subsection{XPS/UPS}

X-ray photoelectron spectroscopy (XPS) and Ultraviolet photoelectron spectroscopy (UPS) are used to measure information of a surface ( $\sim 10 \mathrm{~nm}$ for XPS and $\sim 2-5 \mathrm{~nm}$ for UPS), including elemental composition, chemical states of the element, electronic structure of the valence states, and work function ${ }^{8,102-105}$. A common measurement process for XPS and UPS is shown in Figure 4.8. A beam of X-ray/Ultraviolet radiation with a known energy irradiates a sample surface and is absorbed by electrons of various energy levels. The excited electrons have an energy higher than their binding energy in the materials, such that they are emitted from the surface of the material with a certain kinetic energy. The kinetic energies of those photo-electrons are detected and recorded by a detector. 


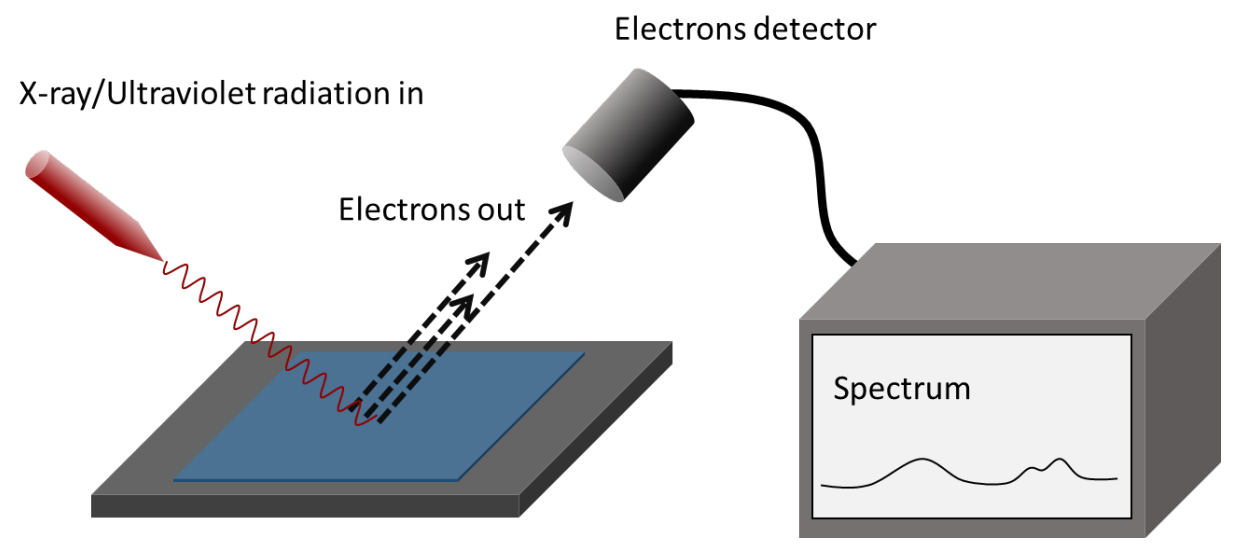

Figure 4.8 Schematic of basic components of a XPS/UPS system.

The binding energy of these electrons can be obtained by:

$$
E_{b}=E_{p}-\left(E_{k}+\phi\right)
$$

where $E_{b}$ is the electron binding energy, $E_{p}$ is the photon energy from radiation source with a particular wavelength, $E_{k}$ is the measured emitted electron kinetic energy, $\phi$ is the work function.

The electronic structure of a material is made of electrons that are localized around the nuclei, which are called core-electrons, and electrons delocalized around several atoms and involved in bonds, that are called valence-electrons. Each atom is thus characterized by core electrons that have a specific binding energy. Hence, by looking at the peak in an XPS spectrum, we can identify the type of atoms present and their concentration. Moreover, depending on the electronic density of the valence electron on an atom, the binding energy of the core electrons will be affected. For instance, in polyethylene terephthalate, there are several carbons. The binding energy of the $1 \mathrm{~s}-$ core electron of carbon atoms is around 280-290 eV. But looking at the C(1s) spectra, we see three different peaks. A carbon atom attached to two oxygen atoms will have a high binding energy $(289 \mathrm{eV})$. Indeed, two electronegative atoms are pumping the valence electron density around the carbon atom, such that the core-electrons of the carbon atom are more attracted by the positively charged nucleus due to the poor 
valence electron density. In contrast, a carbon atom surrounded of other carbon atoms of same electronegativity will have a lower binding energy since there is a high valence electron density around it to screen the positive charge of the nucleus.

In this thesis, to study the reaction between PEDOT:PSS and GOPS, the chemical composition of film specimens were measured by XPS and UPS. UPS result together with Kelvin Probe measurements showed that the work function is not changing for PEDOT:PSS films with different ratio of GOPS. XPS results helped us to identify the chemical reaction and new cross-linking bonds formed between GOPS and the $-\mathrm{SO}_{3}{ }^{-}$ groups of PSS. 


\subsection{Evaporation rate}

Evaporation rate with a unit of $\mathrm{kg} \mathrm{m}^{-2} \mathrm{~h}^{-1}$ is the most important property of a water steam generator ${ }^{26,106-108}$. It is related to many factors including the water surface area, temperature of water and environment, light, and airflow. From the point of view of solar absorber materials, all of the other factors except materials should be constant. A water evaporation rate measurement is usually done in lab environment, with room temperature, no airflow, and with 1 -sun $\left(1 \mathrm{~kW} \mathrm{~m}^{-2}\right)$ irradiation. A common measurement setup is shown in Figure 4.9.

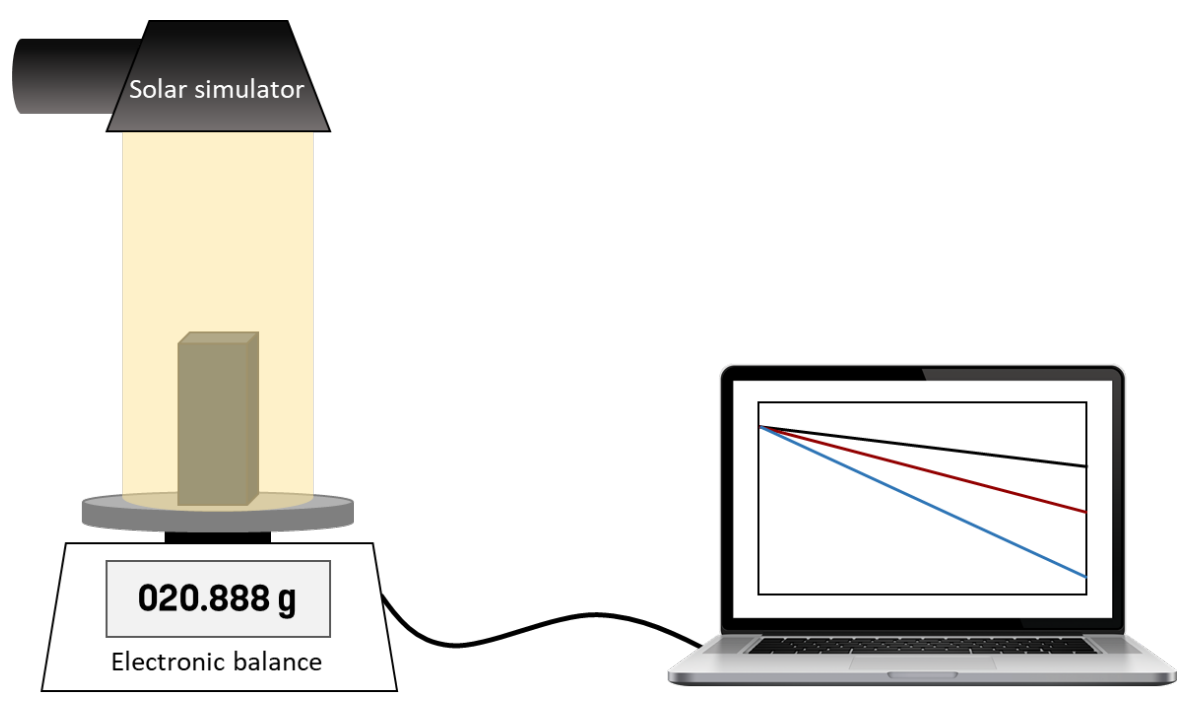

Figure 4.9 Schematic of a water evaporation rate measurement setup. 


\section{Conclusion and Outlook}

\subsection{Overview of publications}

Paper 1: Effect of (3-Glycidyloxypropyl)Trimethoxysilane (GOPS) on the Electrical Properties of PEDOT:PSS Films

GOPS was used as a cross linker in order to improve the mechanical stability of PEDOT:PSS in aqueous solution. We studied the relationship between the electrical conductivity reduction of the PEDOT composite and the amount of GOPS addition. We proposed a mechanism to explain the chemical crosslinking reaction between GOPS and PEDOT:PSS. We studied the electrical conductivity, absorbance spectrum, work function and morphology changing with different ratios of GOPS. It is found that the epoxy ring of GOPS reacts with the sulfonate group of PSS, and the methoxysilane group on another side of GOPS reacts between GOPS molecules.

Paper 2: Thermoelectric Polymer Aerogels for Pressure-Temperature Sensing Applications

In this work, we conveyed the thermoelectric and semimetallic properties of PEDOT:PSS into a three-dimensional scaffold by means of a unique combination of nanocellulose and conducting polymers, to create thermoelectric polymer aerogels. The addition of GOPS in this aerogel changed its mechanical property. Indeed, intrinsically without GOPS, the aerogel was fragile and brittle, but the introduction of 
GOPS made it elastic. We discovered that the transport of charge carriers in these aerogels is free of activation barrier when they are exposed to high boiling point solvents, thus reaching the transition between insulator to semimetal behavior. These thermoelectric polymer aerogels were used as ideal dual parameter pressure and temperature sensors. Indeed, the reading of the electric signal was free of cross-talking, that is the reading of pressure and temperature is independent from each other. The success of this dual pressure-temperature sensors is due to the specific activation free transport that enables the reading of pressure and temperature without any cross talk.

\section{Paper 3: A Multiparameter Pressure-Temperature-Humidity Sensor Based on Mixed Ionic-Electronic Cellulose Aerogels}

This is an extension of the work in Paper 2, where we took the challenge to see if yet another parameter, the humidity level, could be detected independently from pressure and temperature. We had to improve the ionic conductivity to get the humidity sensing mechanism and we had to redesign the aerogel shape in the sensor device. The mixed ionic-electronic conducing properties of the aerogels was the key for this threeparameter sensor. The aerogel is highly porous and conducts both electrons and ions simultaneously. Moreover, the aerogel is elastic and changes resistance upon pressure. We use the combination of electronic Seebeck effect and ionic Seebeck effect to access sensing of both humidity and temperature. We could disentangle them by measuring open voltage versus time. Therefore, the three parameters (pressure, temperature, and humidity) can be detected and transformed into three different electronic signals separately without crosstalk (in a specific range of temperature, pressure and humidity). As a cross linker, GOPS was used in this work to enhance both the elasticity of the aerogel and the water stability of PEDOT:PSS in high humidity environment.

\section{Paper 4: Solar Steam Generation Enabled by Cellulose Aerogels}

In this work, we report a cellulose based solar vapor generation system which uses a PEDOT:PSS/NFC aerogel as the solar absorber and water evaporator, with an expanded polyethylene (EPE) foam as the thermal insulator. The solar energy of 1 sun can be almost completely absorbed and converted into thermal energy by the aerogel, 
with the thermal energy mostly located in the aerogel. The freeze-dried aerogel has micro-scale channels that allow water to penetrate into the solar absorber, while providing a large specific surface area for water evaporation. The rough surface of the channel walls further increases the internal surface area for evaporation. The rapid water diffusion and capillary pumping of the micro-scale channels lead to rapid replenishment of the internal surface area to support a sustained high evaporation rate.

\subsection{Conclusion}

In this thesis, a novel composite that combined organic thermoelectric material and cellulose based aerogel was designed and fabricated and two applications, sensing and water treatment, were studied. The two applications require the material to be stable in high humidity environment and in water. As a cross linker, GOPS was used to enable water stability of PEDOT:PSS aerogels and the mechanism of the reaction between GOPS and PEDOT:PSS was investigated. The sulfonate group on PSS chain reacts with one side of the GOPS and generates a new chemical bond; the other side of the GOPS reacts with another GOPS molecule and hence a network is established. The water stability is increased because of the reducing of sulfonate group on PSS and the presence of network with GOPS.

A 3-D porous aerogel was established by PEDOT:PSS, GOPS, and nano cellulose. The potential of the aerogel as a multi parameter sensor material was studied, as a goal of this thesis was to contribute for simplifying the manufacturing of sensors by using the multifunction of new materials. The nanofiber of cellulose and GOPS are offering the mechanical properties, the PEDOT provides the electronic conductivity and electronic Seebeck response, the PSS provides the ionic conductivity and ionic Seebeck response. In this thesis, we focused the three key physical parameters needed in many contexts: pressure, temperature, and humidity. The skin of our body is designed to be sensitive to those parameters too, hence such sensors can likely have an interest in robotics and electronic skins. The strategy was to sense pressure by reading the resistance, to sense temperature by reading the Seebeck voltage, and to sense humidity with ionic thermo- 
induced voltage. A challenge for a multi-parameter sensor is to eliminate the mutual interferences between different signals. We have found that that a high boiling point solvent such as DMSO can change the charge carrier transport of PEDOT:PSS and hence the resistance of an aerogel is no longer changing with temperature. As a result, pressure and temperature signals can be detected by an aerogel without cross talk. Beside electronic conductor, PEDOT:PSS is also a good ionic conductor. The ionic thermoelectric performance of PEDOT:PSS is a function of environmental humidity, therefore, the humidity can be extracted from a measuring of the evolving Seebeck voltage.

The cellulose-PEDOT based porous aerogel was used as a solar energy absorber, which converts the energy into heat and drives the water molecules evaporate from the source salted water. The cellulose and GOPS ensure that the porous aerogel is not collapsing in water and the pores remain open to promote the water evaporation. The PEDOT enables the absorption of light in both visible and infrared ranges and its conversion into heat. Indeed, the presence of quasi-free electron in PEDOT quenches efficiently the exciton so that no fluorescence take place. The resulting aerogel appears dark blue. A $2 \mathrm{~mm}$ thick aerogel can absorb more than $99 \%$ of the solar energy and hence provide heat to evaporate water. The porous structure of the aerogel enabled water molecules to have high interface area for evaporating. By localizing the heat in the aerogel, evaporation rate of a solar steam generation system was reached to 1.61 $\mathrm{kg} \mathrm{m}^{-2} \mathrm{~h}^{-1}$ at 1 sun irradiation, with a conversion efficiency of $81 \%$.

\subsection{Outlook}

Here we discuss a few directions that could be further exploited to improve the performance of the materials for various applications. The use of the silane crosslinker GOPS increased the elasticity and water stability of both cellulose and PEDOT:PSS. It could be interesting to understand the interaction of cellulose with GOPS and the origin of its elastic character. The use of GOPS with PEDOT:PSS has of course the drawback to decrease its electrical conductivity since GOPS is an insulator. However, 
other studies indicated that composites made of polymer semiconductor and polymer insulator can reach high mobility if a proper morphology is created inside the blend. Hence, it is not excluded that other crosslinking material could not be designed with less detrimental effects on electrical properties.

Ionic Seebeck effect of PSS and mixed conductor PEDOT:PSS has not been thoroughly studied. The nature of the cations, the nature of the ion channel in the polymer, the size of the cations and its electrochemical activity are several parameters that could be investigated to optimize the response of the sensor to humidity. A larger ionic Seebeck coefficient and a faster response would be favorable for the sensor.

We have not finished a systematically investigate on the effect of the porosity of the aerogels on the many properties relevant for the sensors and solar evaporators. There are many factors that can affect the properties of the aerogels, including the recipe and manufacturing process. By varying these factors, aerogels with different properties (e.g. density, porous size, electrical properties, and mechanical properties) can be produced in a more systematic fashion.

For solar steam generation application, we have used PEDOT as infrared absorber but it would be interesting to add other polymer dyes absorbing more in the visible to see if the efficiency of light-to-heat conversion can be improved. Although the cost of the cellulose is low, the manufacturing cost of the aerogels is relatively high because of the freeze-drying process (energy intensive), which might prevent scaling up into large scale solar evaporator. With the premise of performance, it will be a valuable study direction to find alternative manufacturing process. In this context, our colleagues found that classical freezing at $-10^{\circ} \mathrm{C}$ can be used to induce crosslinking in cellulose, a process that could be used to create aerogels ${ }^{96}$. 


\section{References}

1 Kemerink, M., Timpanaro, S., de Kok, M. M., Meulenkamp, E. A. \& Touwslager, F. J. Three-dimensional inhomogeneities in PEDOT : PSS films. J Phys Chem B 108, 18820-18825, doi:DOI 10.1021/jp0464674 (2004).

2 Yan, H. et al. Poly(3,4-ethylenedioxythiophene)/poly(4-styrenesulfonate): Correlation between colloidal particles and thin films. Thin Solid Films 517, 3299-3303, doi:10.1016/j.tsf.2009.01.004 (2009).

3 Yan, H., Jo, T. \& Okuzaki, H. Highly Conductive and Transparent Poly(3,4ethylenedioxythiophene)/Poly(4-styrenesulfonate) (PEDOT/PSS) Thin Films. Polym J 41, 1028-1029, doi:10.1295/polymj.PJ2009143 (2009).

4 Bubnova, O. et al. Optimization of the thermoelectric figure of merit in the conducting polymer poly(3,4-ethylenedioxythiophene). Nature Materials 10, 429-433, doi:10.1038/Nmat3012 (2011).

5 Lipomi, D. J. et al. Skin-like pressure and strain sensors based on transparent elastic films of carbon nanotubes. Nature Nanotechnology 6, 788-792, doi:10.1038/Nnano.2011.184 (2011).

6 Sahatiya, P. \& Badhulika, S. Eraser-based eco-friendly fabrication of a skin-like large-area matrix of flexible carbon nanotube strain and pressure sensors. Nanotechnology 28, doi:10.1088/1361-6528/aa5845 (2017).

7 Hammock, M. L., Chortos, A., Tee, B. C. K., Tok, J. B. H. \& Bao, Z. A. 25th Anniversary Article: The Evolution of Electronic Skin (E-Skin): A Brief History, Design Considerations, and Recent Progress. Advanced Materials 25, 59976037, doi:10.1002/adma.201302240 (2013).

8 Malti, A. et al. An Organic Mixed Ion-Electron Conductor for Power Electronics. Adv Sci 3, doi:10.1002/advs.201500305 (2016).

9 Tritt, T. M. \& Subramanian, M. A. Thermoelectric materials, phenomena, and applications: A bird's eye view. Mrs Bull 31, 188-194, doi:DOI 10.1557/mrs2006.44 (2006).

10 Sootsman, J. R., Chung, D. Y. \& Kanatzidis, M. G. New and Old Concepts in Thermoelectric Materials. Angew Chem Int Edit 48, 8616-8639, doi:10.1002/anie.200900598 (2009).

11 Dresselhaus, M. S. et al. New directions for low-dimensional thermoelectric materials. Advanced Materials 19, 1043-1053, doi:10.1002/adma.200600527 (2007).

12 Kistler, S. S. Coherent expanded aerogels adn jellies. Nature 127, doi:10.1038/127741a0 (1931). 
13 Maleki, H. et al. Synthesis and biomedical applications of aerogels: Possibilities and challenges. Adv Colloid Interface Sci 236, 1-27, doi:10.1016/j.cis.2016.05.011 (2016).

14 He, W. \& Zhang, X. in Conducting Polymers Ch. Chapter 3, (2016).

15 Feng, J. et al. Silica cellulose hybrid aerogels for thermal and acoustic insulation applications. Colloids and Surfaces A: Physicochemical and Engineering Aspects 506, 298-305, doi:10.1016/j.colsurfa.2016.06.052 (2016).

16 Hees, T., Zhong, F., Rudolph, T., Walther, A. \& Mülhaupt, R. Nanocellulose Aerogels for Supporting Iron Catalysts and In Situ Formation of Polyethylene Nanocomposites. Advanced Functional Materials 27, doi:10.1002/adfm.201605586 (2017).

17 Plata, D. L. et al. Aerogel-platform optical sensors for oxygen gas. Journal of Non-Crystalline Solids 350, 326-335, doi:10.1016/j.jnoncrysol.2004.06.046 (2004).

18 Wu, Z. Y. et al. Carbon nanofiber aerogels for emergent cleanup of oil spillage and chemical leakage under harsh conditions. Sci Rep-Uk 4, doi:10.1038/srep04079 (2014).

19 Nystrom, G. et al. Self-assembled three-dimensional and compressible interdigitated thin-film supercapacitors and batteries. Nature Communications 6, doi:10.1038/ncomms8259 (2015).

20 Jiang, F. et al. Lightweight, Mesoporous, and Highly Absorptive All-Nanofiber Aerogel for Efficient Solar Steam Generation. ACS Appl Mater Interfaces 10, 1104-1112, doi:10.1021/acsami.7b15125 (2018).

$21 \mathrm{He}, \mathrm{W}$. et al. Polypyrrole/silver coaxial nanowire aero-sponges for temperature-independent stress sensing and stress-triggered Joule heating. Acs Nano 9, 4244-4251, doi:10.1021/acsnano.5b00626 (2015).

22 Bryning, M. B. et al. Carbon Nanotube Aerogels. Advanced Materials 19, 661664, doi:10.1002/adma.200601748 (2007).

23 Khan, Z. U. et al. Thermoelectric Polymers and their Elastic Aerogels. Adv Mater 28, 4556-4562, doi:10.1002/adma.201505364 (2016).

24 Miorandi, D., Sicari, S., De Pellegrini, F. \& Chlamtac, I. Internet of things: Vision, applications and research challenges. Ad Hoc Networks 10, 1497-1516, doi:10.1016/j.adhoc.2012.02.016 (2012).

25 Li, S. C., Xu, L. D. \& Zhao, S. S. The internet of things: a survey. Inform Syst Front 17, 243-259, doi:10.1007/s10796-014-9492-7 (2015).

$26 \mathrm{Hu}, \mathrm{X} . \mathrm{Z}$. et al. Tailoring Graphene Oxide-Based Aerogels for Efficient Solar Steam Generation under One Sun. Advanced Materials 29, doi:10.1002/adma.201604031 (2017). 
27 Bae, K. et al. Flexible thin-film black gold membranes with ultrabroadband plasmonic nanofocusing for efficient solar vapour generation. Nature Communications 6, doi:10.1038/ncomms10103 (2015).

28 Zhu, M. W. et al. Plasmonic Wood for High-Efficiency Solar Steam Generation. Adv Energy Mater 8, doi:10.1002/aenm.201701028 (2018).

29 Groenendaal, B. L., Jonas, F., Freitag, D., Pielartzik, H. \& Reynolds, J. R. Poly(3,4-ethylenedioxythiophene) and its derivatives: Past, present, and future. Advanced Materials 12, 481-494 (2000).

30 Shiraishi, K., Kanbara, T., Yamamoto, T. \& Groenendaal, L. Preparation of a soluble and neutral alkyl derivative of poly(3,4-ethylenedioxythiophene) and its optical properties. Polymer 42, 7229-7232, doi:Doi 10.1016/S00323861(01)00137-9 (2001).

31 Bubnova, O. et al. Semi-metallic polymers. Nat Mater 13, 190-194, doi:10.1038/nmat3824 (2014).

32 Nevrela, J. et al. Secondary doping in poly(3,4ethylenedioxythiophene):Poly(4-styrenesulfonate) thin films. J Polym Sci Pol Phys 53, 1139-1146, doi:10.1002/polb.23754 (2015).

33 Macdiarmid, A. G. \& Epstein, A. J. Secondary Doping in Polyaniline. Synthetic Metals 69, 85-92, doi:Doi 10.1016/0379-6779(94)02374-8 (1995).

34 Donoval, M. et al. Relation between secondary doping and phase separation in PEDOT:PSS films. Appl Surf Sci 395, 86-91, doi:10.1016/j.apsusc.2016.05.076 (2017).

35 Jia, C. et al. From Wood to Textiles: Top-Down Assembly of Aligned Cellulose Nanofibers. Advanced Materials 30, doi:10.1002/adma.201801347 (2018).

36 Dutta, S. et al. 3D network of cellulose-based energy storage devices and related emerging applications. Mater Horiz 4, 522-545, doi:10.1039/c6mh00500d (2017).

37 Wang, X. D., Yao, C. H., Wang, F. \& Li, Z. D. Cellulose-Based Nanomaterials for Energy Applications. Small 13, doi:10.1002/smll.201702240 (2017).

38 Wang, S., Lu, A. \& Zhang, L. N. Recent advances in regenerated cellulose materials. Prog Polym Sci 53, 169-206, doi:10.1016/j.progpolymsci.2015.07.003 (2016).

39 Carpenter, A. W., de Lannoy, C. F. \& Wiesner, M. R. Cellulose Nanomaterials in Water Treatment Technologies. Environ Sci Technol 49, 5277-5287, doi:10.1021/es506351r (2015).

40 Bethke, K. et al. Functionalized Cellulose for Water Purification, Antimicrobial Applications, and Sensors. Advanced Functional Materials 28, doi:0.1002/adfm.201800409 (2018). 
41 Trache, D., Hussin, M. H., Haafiz, M. K. M. \& Thakur, V. K. Recent progress in cellulose nanocrystals: sources and production. Nanoscale 9, 1763-1786, doi:10.1039/c6nr09494e (2017).

42 Siro, I. \& Plackett, D. Microfibrillated cellulose and new nanocomposite materials: a review. Cellulose 17, 459-494, doi:10.1007/s10570-010-9405-y (2010).

43 Klemm, D., Heublein, B., Fink, H. P. \& Bohn, A. Cellulose: Fascinating biopolymer and sustainable raw material. Angew Chem Int Edit 44, 33583393, doi:10.1002/anie.200460587 (2005).

44 Rinaldi, R. et al. Paving the Way for Lignin Valorisation: Recent Advances in Bioengineering, Biorefining and Catalysis. Angew Chem Int Edit 55, 81648215, doi:10.1002/anie.201510351 (2016).

$45 \mathrm{Kai}, \mathrm{D}$. et al. Towards lignin-based functional materials in a sustainable world. Green Chem 18, 1175-1200, doi:10.1039/c5gc02616d (2016).

46 Jonoobi, M. et al. Different preparation methods and properties of nanostructured cellulose from various natural resources and residues: a review. Cellulose 22, 935-969, doi:10.1007/s10570-015-0551-0 (2015).

47 Saito, T., Kimura, S., Nishiyama, Y. \& Isogai, A. Cellulose nanofibers prepared by TEMPO-mediated oxidation of native cellulose. Biomacromolecules 8 , 2485-2491, doi:10.1021/bm0703970 (2007).

48 Wagberg, L. et al. The build-up of polyelectrolyte multilayers of microfibrillated cellulose and cationic polyelectrolytes. Langmuir $\mathbf{2 4 , 7 8 4 - 7 9 5 , ~}$ doi:10.1021/la702481v (2008).

49 Elsheikh, M. H. et al. A review on thermoelectric renewable energy: Principle parameters that affect their performance. Renew Sust Energ Rev 30, 337-355, doi:10.1016/j.rser.2013.10.027 (2014).

50 Fan, Z., Du, D., Yao, H. \& Ouyang, J. Higher PEDOT Molecular Weight Giving Rise to Higher Thermoelectric Property of PEDOT:PSS: A Comparative Study of Clevios P and Clevios PH1000. ACS Appl Mater Interfaces 9, 11732-11738, doi:10.1021/acsami.6b15158 (2017).

51 Petsagkourakis, l. et al. Correlating the Seebeck coefficient of thermoelectric polymer thin films to their charge transport mechanism. Org Electron 52, 335341, doi:10.1016/j.orgel.2017.11.018 (2018).

52 Burger, N. et al. Review of thermal conductivity in composites: Mechanisms, parameters and theory. Prog Polym Sci 61, 1-28, doi:10.1016/j.progpolymsci.2016.05.001 (2016).

53 Choy, C. L. Thermal-Conductivity of Polymers. Polymer 18, 984-1004, doi:Doi 10.1016/0032-3861(77)90002-7 (1977). 
54 Petsagkourakis, I. et al. Thermoelectric materials and applications for energy harvesting power generation. Sci Technol Adv Mat 19, 836-862, doi:10.1080/14686996.2018.1530938 (2018).

55 Michalski, J. Thermal-Conductivity of Amorphous Solids above the Plateau Molecular-Dynamics Study. Phys Rev B 45, 7054-7065, doi:DOI 10.1103/PhysRevB.45.7054 (1992).

56 Wang, S. H. et al. Effect of Backbone Regiochemistry on Conductivity, Charge Density, and Polaron Structure of n-Doped Donor-Acceptor Polymers. Chem Mater 31, 3395-3406, doi:10.1021/acs.chemmater.9b00558 (2019).

57 Zozoulenko, I. et al. Polarons, Bipolarons, And Absorption Spectroscopy of PEDOT. ACS Applied Polymer Materials 1, 83-94, doi:10.1021/acsapm.8b00061 (2018).

58 Kim, D. \& Zozoulenko, I. Why Is Pristine PEDOT Oxidized to 33\%? A Density Functional Theory Study of Oxidative Polymerization Mechanism. J Phys Chem B 123, 5160-5167, doi:10.1021/acs.jpcb.9b01745 (2019).

59 Munoz, W. A. et al. Insulator to semimetallic transition in conducting polymers. Phys Rev B 94, doi:10.1103/PhysRevB.94.205202 (2016).

$60 \mathrm{Kim}, \mathrm{G}$. \& Pipe, K. P. Thermoelectric model to characterize carrier transport in organic semiconductors. Phys Rev B 86, doi:10.1103/PhysRevB.86.085208 (2012).

61 Troisi, A. Charge transport in high mobility molecular semiconductors: classical models and new theories. Chem Soc Rev 40, 2347-2358, doi:10.1039/c0cs00198h (2011).

62 Sirringhaus, H. et al. Two-dimensional charge transport in self-organized, high-mobility conjugated polymers. Nature 401, 685-688, doi:Doi 10.1038/44359 (1999).

63 Noriega, R. et al. A general relationship between disorder, aggregation and charge transport in conjugated polymers. Nature Materials 12, 1038-1044, doi:10.1038/nmat3722 (2013).

64 Wang, S. H. et al. Experimental evidence that short-range intermolecular aggregation is sufficient for efficient charge transport in conjugated polymers. Proceedings of the National Academy of Sciences of the United States of America 112, 10599-10604, doi:10.1073/pnas.1501381112 (2015).

65 Lee, K. et al. Metallic transport in polyaniline. Nature 441, 65-68, doi:10.1038/nature04705 (2006).

66 Chen, S. Z. et al. On the anomalous optical conductivity dispersion of electrically conducting polymers: ultra-wide spectral range ellipsometry combined with a Drude-Lorentz model. J Mater Chem C 7, 4350-4362, doi:10.1039/c8tc06302h (2019). 
67 Palumbiny, C. M. et al. Molecular Reorientation and Structural Changes in Cosolvent-Treated Highly Conductive PEDOT:PSS Electrodes for Flexible Indium Tin Oxide-Free Organic Electronics. J Phys Chem C 118, 13598-13606, doi:10.1021/jp501540y (2014).

68 Ouyang, J. et al. On the mechanism of conductivity enhancement in poly $(3,4-$ ethylenedioxythiophene): poly(styrene sulfonate) film through solvent treatment. Polymer 45, 8443-8450, doi:10.1016/j.polymer.2004.10.001 (2004).

69 Lee, I., Kim, G. W., Yang, M. \& Kim, T. S. Simultaneously Enhancing the Cohesion and Electrical Conductivity of PEDOT:PSS Conductive Polymer Films using DMSO Additives. Acs Appl Mater Inter 8, 302-310, doi:10.1021/acsami.5b08753 (2016).

70 Timpanaro, S., Kemerink, M., Touwslager, F. J., De Kok, M. M. \& Schrader, S. Morphology and conductivity of PEDOT/PSS films studied by scanningtunneling microscopy. Chemical Physics Letters 394, 339-343, doi:10.1016/j.cplett.2004.07.035 (2004).

71 Thomas, J. P., Zhao, L. Y., McGillivray, D. \& Leung, K. T. High-efficiency hybrid solar cells by nanostructural modification in PEDOT:PSS with co-solvent addition. Journal of Materials Chemistry A 2, 2383-2389, doi:10.1039/c3ta14590e (2014).

72 Rivnay, J. et al. Structural control of mixed ionic and electronic transport in conducting polymers. Nature Communications 7, doi:10.1038/ncomms11287 (2016).

73 A. G. MacDiarmid, A. J. E. The concept of secondary doping as applied to polyaniline. Synthetic Metals 65 (1994).

74 Crispin, X. et al. The origin of the high conductivity of poly(3,4ethylenedioxythiophene)-poly(styrenesulfonate) (PEDOT-PSS) plastic electrodes. Chem Mater 18, 4354-4360, doi:10.1021/cm061032+ (2006).

75 Nardes, A. M., Janssen, R. A. J. \& Kemerink, M. A morphological model for the solvent-enhanced conductivity of PEDOT : PSS thin films. Advanced Functional Materials 18, 865-871, doi:10.1002/adfm.200700796 (2008).

76 Kim, B. et al. Robust High Thermoelectric Harvesting Under a Self-Humidifying Bilayer of Metal Organic Framework and Hydrogel Layer. Advanced Functional Materials 29, doi:10.1002/adfm.201807549 (2019).

77 Paulsen, B. D., Tybrandt, K., Stavrinidou, E. \& Rivnay, J. Organic mixed ionicelectronic conductors. Nat Mater, doi:10.1038/s41563-019-0435-z (2019).

78 Stavrinidou, E. et al. Direct measurement of ion mobility in a conducting polymer. Adv Mater 25, 4488-4493, doi:10.1002/adma.201301240 (2013). 
79 Kus, M. \& Okur, S. Electrical characterization of PEDOT:PSS beyond humidity saturation. Sensor Actuat B-Chem 143, 177-181, doi:10.1016/j.snb.2009.08.055 (2009).

80 Wang, H., Ail, U., Gabrielsson, R., Berggren, M. \& Crispin, X. lonic Seebeck Effect in Conducting Polymers. Adv Energy Mater 5, doi:10.1002/aenm.201500044 (2015).

81 Stefan Duhr, D. B. Why molecules move along a temperature gradient. Proceedings of the National Academy of Sciences of the United States of America 103, doi:https://doi.org/10.1073/pnas.0603873103 (2006).

82 Ail, U. et al. Thermoelectric Properties of Polymeric Mixed Conductors. Advanced Functional Materials 26, 6288-6296, doi:10.1002/adfm.201601106 (2016).

83 Zhao, D. et al. Polymer gels with tunable ionic Seebeck coefficient for ultrasensitive printed thermopiles. Nature Communications 10, doi:10.1038/s41467-019-08930-7 (2019).

84 Wang, H. et al. Ionic Thermoelectric Figure of Merit for Charging of Supercapacitors. Advanced Electronic Materials 3, doi:10.1002/aelm.201700013 (2017).

85 Zhao, D. et al. Ionic thermoelectric supercapacitors. Energ Environ Sci 9, 14501457, doi:10.1039/c6ee00121a (2016).

86 Scholes, G. D., Fleming, G. R., Olaya-Castro, A. \& van Grondelle, R. Lessons from nature about solar light harvesting. Nat Chem 3, 763-774, doi:10.1038/Nchem.1145 (2011).

87 Cheng, P., Li, G., Zhan, X. W. \& Yang, Y. Next-generation organic photovoltaics based on non-fullerene acceptors. Nat Photonics 12, 131-142, doi:10.1038/s41566-018-0104-9 (2018).

88 Aharonovich, I. Clearly identical photons. Nature Nanotechnology 14, 502503, doi:10.1038/s41565-019-0440-z (2019).

89 Massonnet, N. et al. Improvement of the Seebeck coefficient of PEDOT:PSS by chemical reduction combined with a novel method for its transfer using freestanding thin films. J Mater Chem C 2, 1278-1283, doi:10.1039/c3tc31674b (2014).

90 Khan, Z. U. et al. Acido-basic control of the thermoelectric properties of poly(3,4-ethylenedioxythiophene)tosylate (PEDOT-Tos) thin films. J Mater Chem C 3, 10616-10623, doi:10.1039/c5tc01952d (2015).

91 Ghasemi, H. et al. Solar steam generation by heat localization. Nature Communications 5, doi:10.1038/ncomms5449 (2014).

92 Neumann, O. et al. Nanoparticle-Mediated, Light-Induced Phase Separations. Nano Letters 15, 7880-7885, doi:10.1021/acs.nanolett.5b02804 (2015). 
93 Neumann, O. et al. Solar Vapor Generation Enabled by Nanoparticles. Acs Nano 7, 42-49, doi:10.1021/nn304948h (2013).

94 Wang, M. M. et al. Bifunctional plasmonic colloidosome/graphene oxidebased floating membranes for recyclable high-efficiency solar-driven clean water generation. Nano Res 11, 3854-3863, doi:10.1007/s12274-017-1959-7 (2018).

95 Jiang, F. \& Hsieh, Y. L. Amphiphilic superabsorbent cellulose nanofibril aerogels. Journal of Materials Chemistry A 2, 6337-6342, doi:10.1039/c4ta00743c (2014).

96 Erlandsson, J. et al. On the mechanism behind freezing-induced chemical crosslinking in ice-templated cellulose nanofibril aerogels. Journal of Materials Chemistry A 6, doi:10.1039/c8ta06319b (2018).

$97 \mathrm{Hu}$, Y., Acharya, S. \& Abidi, N. Cellulose porosity improves its dissolution by facilitating solvent diffusion. Int J Biol Macromol 123, 1289-1296, doi:10.1016/j.ijbiomac.2018.10.062 (2019).

98 Jimenez-Saelices, C., Seantier, B., Cathala, B. \& Grohens, Y. Spray freeze-dried nanofibrillated cellulose aerogels with thermal superinsulating properties. Carbohyd Polym 157, 105-113, doi:10.1016/j.carbpol.2016.09.068 (2017).

99 Schuetze, A. P., Lewis, W., Brown, C. \& Geerts, W. J. A laboratory on the fourpoint probe technique. Am J Phys 72, 149-153, doi:10.1119/1.1629085 (2004).

100 Trznadel, M. et al. UV-VIS-NIR and Raman spectroelectrochemistry of regioregular poly(3-octylthiophene): Comparison with its non-regioregular analogue. J Chem Soc Faraday T 92, 1387-1393, doi:DOI 10.1039/ft9969201387 (1996).

101 Yamamoto, J. \& Furukawa, Y. Electronic and vibrational spectra of positive polarons and bipolarons in regioregular poly(3-hexylthiophene) doped with ferric chloride. J Phys Chem B 119, 4788-4794, doi:10.1021/jp512654b (2015).

102 Siokou, A. et al. Surface refinement and electronic properties of graphene layers grown on copper substrate: An XPS, UPS and EELS study. App/ Surf Sci 257, 9785-9790, doi:10.1016/j.apsusc.2011.06.017 (2011).

103 Tada, A., Geng, Y. F., Wei, Q. S., Hashimoto, K. \& Tajima, K. Tailoring organic heterojunction interfaces in bilayer polymer photovoltaic devices. Nature Materials 10, 450-455, doi:10.1038/Nmat3026 (2011).

104 Hintz, H., Peisert, H., Egelhaaf, H. J. \& Chasse, T. Reversible and Irreversible Light-Induced $\mathrm{p}$-Doping of P3HT by Oxygen Studied by Photoelectron Spectroscopy (XPS/UPS). J Phys Chem C 115, 13373-13376, doi:10.1021/jp2032737 (2011). 
105 Fabiano, S. et al. Poly(ethylene imine) Impurities Induce n-doping Reaction in Organic (Semi)Conductors. Advanced Materials 26, 6000-+, doi:10.1002/adma.201401986 (2014).

$106 \mathrm{Xu}, \mathrm{N}$. et al. Mushrooms as Efficient Solar Steam-Generation Devices. Advanced Materials 29, doi:10.1002/adma.201606762 (2017).

107 Zhou, L. et al. Self-assembly of highly efficient, broadband plasmonic absorbers for solar steam generation. Sci Adv 2, doi:10.1126/sciadv.1501227 (2016).

108 Jiang, Q. S. et al. Bilayered Biofoam for Highly Efficient Solar Steam Generation. Advanced Materials 28, 9400-+, doi:10.1002/adma.201601819 (2016). 



\section{Papers}

The papers associated with this thesis have been removed for copyright reasons. For more details about these see:

http://urn.kb.se/resolve?urn=urn:nbn:se:liu:diva-161350 


\section{FACULTY OF SCIENCE AND ENGINEERING}

Linköping Studies in Science and Technology, Dissertation No. 2028, 2019

Department of Science and Technology (ITN)

Linköping University

SE-581 83 Linköping, Sweden

www.liu.se 